\title{
A Study of Visual Perception in Fish, Especially on Resolving Power and Accommodation*
}

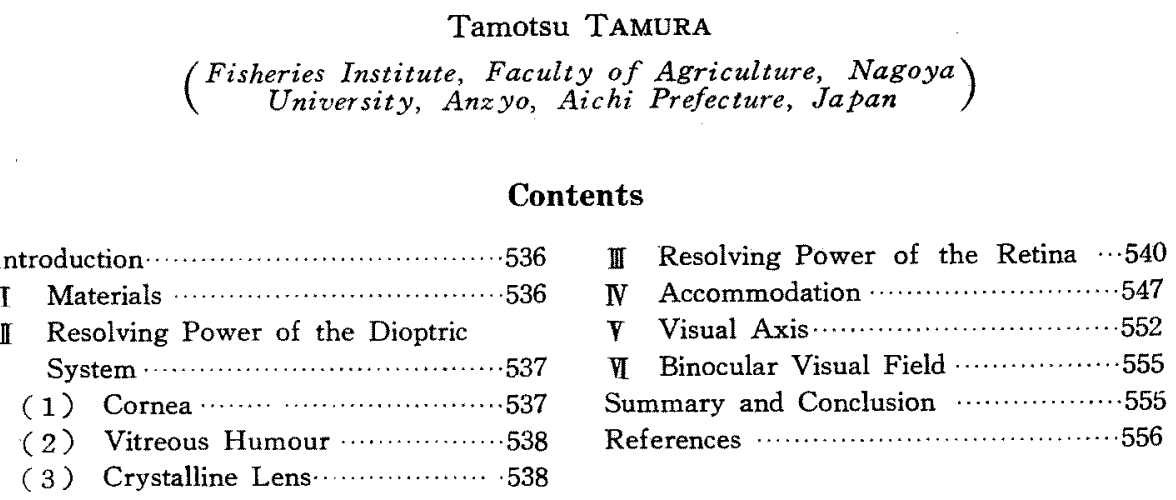

\section{Introduction}

Any object in visual space may have a number of attributes, i.e., form, brightness, colour, motion, etc. Of these, form perception in fish is studied in the present paper. Very little comparative data on form perception is available, even though numerous anatomical and optical studies of the fish have been published.

Ability to perceive form depends on two factors : resolving power of the dioptric system, and resolving power of the retina. The first is a function of both the resolving power of the lens and accommodation. The second is presumably depend on the spacing of cones in the retina. In this paper, therefore, resolving power of the lens, dioptric accommodation, and retinal cone density are reported for 27 species representing 18 families (Table 1).

The visual fields of these species have also been described and compared in terms of binocular angle, visual axis, and topographical differences in cone density.

Much of the work with live fish during the course of this study was carried out at the Fisheries Laboratories on Shinmaiko and Ikawazu in Aichi Prefecture. The author is grateful to Emeritus Prof. Dr. I. Aмemiya and Prof. Dr. Y. Oshima of Tokyo University and to Mr. C.P. O' ConNell, fishery research biologist of South Pacific Fishery Investigations of U.S. Fish and Wildlife Service, for reading the manuscript and for their exceedingly helpful and constructive criticism.

\section{Materials}

The 27 marine teleost species used in this study (Table 1) are supposed to rely heavily on vision in their mode of living. Most of them inhabit either the littoral zone or the

Received Oct. 4, 1956 （印刷費負担）

* Contribution from the Fisheries Institute, Faculty of Agriculture. Nagoya University. Series No. 10. 
Table 1. The species used in this study

\begin{tabular}{|c|c|}
\hline Family & Species \\
\hline Argentinidae & Argentina semifasciata Kishinouye* \\
\hline Chlorophthalmidae & Chlorophthalmus albatrossis Jordan et Starks* \\
\hline Zeidae & Zenion japonicum Kamohara* \\
\hline Scombridae & Scomber japonicus Houttuyn \\
\hline Carangidae & $\begin{array}{l}\text { Seriola quinqueradiata Temminck et Schlegel } \\
\text { Trachurus japonicus (Temminck et Schlegel) }\end{array}$ \\
\hline Leiognathidae & Leiogeathus argenteus Lancépède \\
\hline Apogonidae & Apogon lineatus (Temminck et Schlegel) \\
\hline Priacanthidae & Priacanthus boops (Schneider) \\
\hline Serranidae & $\begin{array}{l}\text { Epinephelus septem fasciatus (Thunberg) } \\
\text { E. chlorostigma (Cuvier et Valenciennes) } \\
\text { Latenlabrax japonicus (Cuvier et Valenciennes) } \\
\text { Malakichthys wakiyae Jordan et Hubbs }\end{array}$ \\
\hline Girellidae & Girella punctata Gray \\
\hline Sparidae & $\begin{array}{l}\text { Pagrosomus major (Temminck et Schlegel) } \\
\text { Sparus hasta Bloch et Schneider } \\
\text { S. swinhonis Günther } \\
\text { Evynnis japonicus Tanaka }\end{array}$ \\
\hline Theraponidae & Therapon oxyrhynchus Temminck et Schlegel \\
\hline Hepatidae & Xesurus scalprum Jordan et Fowler \\
\hline Monocanthidae & Cantherines modestus (Günther) \\
\hline Tetraodontidae & $\begin{array}{l}\text { Sphaeroides chrysops (Hilgendorf) } \\
\text { S. niphnbles Jordan et Snyder }\end{array}$ \\
\hline Scorpaenidae & $\begin{array}{l}\text { Sebastiscus marmoratus (Cuvier et Valenciennes) } \\
\text { Helicolenus dactylopterus (De La Roche)* }\end{array}$ \\
\hline Cottidae & Pseudoblennius totomius Jordan et Starks \\
\hline Triglidae & Chelidonichthys kumu (Lesson et Garnot) \\
\hline
\end{tabular}

surface waters of the ocean where there is abundant light. Those marked with asterisks, however, are species with large eyes that are sometimes taken at depths of 100 to 300 meters by deep-sea drag nets.

\section{Resolving Power of the Dioptric System}

Incident light from an object passes through the cornea, anterior chamber, lens and vitreous humour, in that order, to from an image on the retina. The sharpness of this image, which depends on certain properties of the above-named structures, is a measure of the resolving power of the dioptric system. The function of each of these structures will be discussed below.

\section{(1) Cornea}

The cornea of the fish eye is typically a convex, transparent membrane, shaped like the bowl of a teaspoon, the horizontal curvature usually being greater than the vertical curvature (Table 2). According to Walls (1942) this dual curvature is an adaptation of streamling to swift swimming. It does not produce astigmatism, however, because the refractive index of fish-eye cornea is 1.3770 to 1.3775 (MATTHIESSEN 1882, YAMAMoto 1931), very close to that of water, 1.33 , and because the refractive index of anterior chamber is almost equal to that of water. 
Table 2. Curvature of cornea in fish

\begin{tabular}{l|c|c|c|c} 
Species & $\begin{array}{c}\text { Body length } \\
(\mathrm{cm})\end{array}$ & $\begin{array}{c}\text { Diameter of lens } \\
(\mathrm{mm})\end{array}$ & $\begin{array}{c}\text { Radius of curvature } \\
\text { (mm) }\end{array}$ \\
\hline Pagrosomus major & 19 & 6.8 & 13.2 & 8.8 \\
Lateolabrax japonicus & 17 & 6.2 & 11.0 & 6.4 \\
Therapon oxyrhynchus & 19.5 & 6.3 & 10.1 & 6.5 \\
Priacanthus boops & 20 & 12.2 & 17.0 & 16.0 \\
Parapristipoma L. eye & 10 & 4.5 & 7.1 & 4.1 \\
trilineatum R. eye & 11 & 4.5 & 5.2 & 3.8 \\
Sebastiscus marmoratus & 5.5 & 4.4 & 4.1 & 4.0 \\
Sebastodes inermis & 17 & 2.8 & 7.1 & 4.7 \\
Carassius auratus & 4.9 &
\end{tabular}

According to HARMS (1914), some demersal fishes have a protective membrane over the cornea, but these, as well as the adipose lids of such fishes as Mugil and Scomber, are optically similar to the cornea and consequently also optically inert.

\section{(2) Vitreous humour}

The vitreous humour, generally continuous in the posterior and anterior chamber of the fish eye through the aphakic space, also has a refractive index close to that of water, and again plays no part in vision.

\section{(3) Crystalline lens}

The lens is the only dioptric element of the eye, then, that is involved in image formation. Since the lens is spherical and surrounded by media of essentially one refractive index, the principal points and nodal points coincide with the center of the lens.

According to MATthiEssen (1882) and HoGBEN \& LANDGREBe (1940), the optical density is much higher at the center than the periphery of the lens. This graded structure may well serve to reduce spherical aberration and hence improve resolving power.

Resolving power was measured in the following way. The enucleated lens was immersed in physiological salt solution* by attaching its suspensory ligament to a small clip over a transparent container (Fig. 1). A well illuminated movable screen with black and

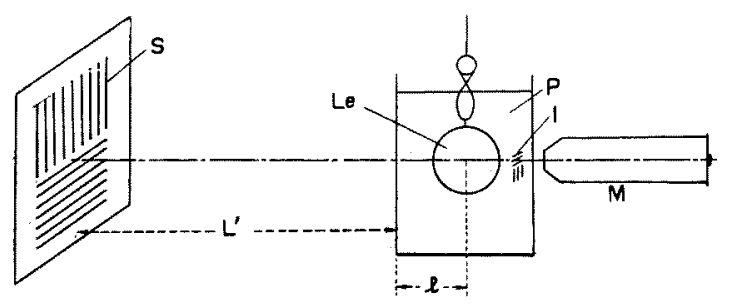

Fig. 1. The method of measuring the resolving power of the lens. Le : Lens, $M$ : Microscope, $S: 1 \mathrm{~mm}$ stripes, P : Physiological salt solution, I : Image of the $1 \mathrm{~mm}$ stripes. white stripes both of which were one millimeter and drawn horizontally and vertically, was placed on one side of the container and a horizontal microscope on the opposite side. The image of the screen projected by the lens could then be viewed through the microscope. The distance between the screen and the lens just to be unable to make out the horizontal and ver-

* According to Yamamoto (1949), in marine fish, $\mathrm{NaCl} 1.35 \%, \mathrm{KCl} 0.06 \%, \mathrm{CaCl}_{2} 0.025 \%$, $\mathrm{MgCl}_{2} 0.035 \%, \mathrm{NaHCO}_{3} 0.002 \%$; in fresh water fish, $\mathrm{NaCl} 0.75 \%, \mathrm{KCl} 0.02 \%, \mathrm{CaCl}_{2} 0.02$ $\%, \quad \mathrm{NaHCO}_{3} 0.002 \%$. 
Table 3. Resolving power of lenses

\begin{tabular}{l|c|c|c}
\hline \multicolumn{1}{|c|}{ Species } & $\begin{array}{c}\text { Diameter of lens } \\
(\mathrm{mm} .)\end{array}$ & $\begin{array}{c}\text { L } \\
(\mathrm{mm} .)\end{array}$ & $\begin{array}{c}\text { Resolving power } \\
(\text { sec.) }\end{array}$ \\
\hline Lateolvbrax japonicus & 8.1 & 3700 & 56 \\
Xesurus scalprum & 7.5 & 3800 & 54 \\
Epinephelus septem fasciatus & 7.3 & 3500 & 59 \\
Sebastiscus marmoratus & 6.2 & 3500 & 59 \\
Cyprinus carpio & 5.4 & 2400 & 86 \\
Lateolabrax japonicus & 4.8 & 2400 & 86 \\
Epinephelus septemfasciatus & 4.7 & 2300 & 90 \\
Gold fish & 1.7 & 1700 & 122 \\
Gold fish & 1.6 & 1700 & 122 \\
Carassius auratus & 1.5 & 1600 & 129 \\
\hline
\end{tabular}

tical stripes of the image could be ascertained by changing the distance L'. The distance in water would be

$$
\mathrm{L}=1.33 \mathrm{~L}^{\prime}+l
$$

where $\mathrm{L}$ is the distance between the object and the lens if this whole experiment were carried out in the water, 1.33 the refractive index of water, $L$ ' the distance from the chamber to the screen, $l$ the distance from the chamber wall to the center of the lens.

The value of $1(\mathrm{~mm}) / \mathrm{L}(\mathrm{mm})=\varphi$ is the resolving power (angle in radians) of the lens. In Table 3 , the values $(\varphi)$ are shown in second of arc.

In ten fishes, the measured values of resolving power range from 54 to 129 seconds of arc, and they seem to have a tendency to become greater in smaller lens. This tendency may partly be attributed to the measuring instrument and the physical property of the light. The factors which affect the values are assumed to be three : the first is a function of the properties of the lens, i.e., the aberrations and the turbidity, the second is the resolving power of the used microscope, and the third is the diffraction of light at the margin of the pupil or the lens. With a large lens the latter two factors are negligible and the measured value is the function of the lens itself; however, with a small lens both factors become too great to be neglected. For example, the image of a one-millimeter stripe projected by a lens $1.5 \mathrm{~mm}$ in diameter (Cararsius) is

$$
I=l \times \frac{F}{L}=\frac{1.91}{1600}=0.0012 \mathrm{~mm}
$$

where $I$ is the image in millimeters, $l$ is the width of the stripe, $L$ is the distance between the object and the lens, and $F$ is the focal distance of the lens as calculated by Matthiessen's ratio, i.e., $F=2.55 \times$ (lens radius). It can readily be seen that the resultant $0.0012 \mathrm{~mm}$ is only slightly greater than the resolving power of the microscope used, which is

$$
d=\frac{\lambda}{2 \mathrm{a}}=\frac{0.00056}{0.50}=0.0011 \mathrm{~mm}
$$

where $d$ is resolving power, $\lambda$ is the wave-length of light and a is the numerical aperture of the microscope. 
Furthermore, the light diffracted at the margin of the pupil whose diameter is the same, or nearly the same, as that of the lens becomes significant enough to effect the resolving power of the lens. Resolving power resulting from such marginal diffraction is as follows for Carassius in Table 3 :

$$
\theta=0.61 \times \frac{\lambda}{r}=0.61 \times \frac{0.00056}{0.75}=94^{\prime \prime}
$$

where $\theta$ is the resolving power resulted from marginal diffraction, 0.61 is the physical constant, $\lambda$ is the wave-length of light, and $r$ is the radius of the pupil. The resultant value, 94 seconds, is only a little less than the measured value of 129 seconds.

Considering the above, the resolving power of lenses of diameter greater than about $5 \mathrm{~mm}$ is $54-90$ seconds. In smaller lenses, it may be assumed to be less than about 120 -130 seconds, though some complications occur in respect to the resolving power of the used microscope and the diffraction of light at the margin of the pupil.

It must be pointed out that the lenses may have greater resolving power in vivo, because they do tend to become turbid while being measured, even though they are in physiological salt solution.

\section{Resolving power of the retina}

The structural basis of resolving power in the retina is the spacing of the cones and the extent to which their conducting neurons are summated. Polyak (1948) has demonstrated that cones in the primate fovea are not summated. Each has a direct nerve tract to the brain. Although very little is known about summation in the teleost retina, it will be assumed, in this report, that cones are not summated.

In fishes, as in all other vertebrate groups, there are two kinds of photoreceptors : rods and cones. Their presence has given rise to the duplicity theory of function. Rods have a low threshold of sensitivity and low acuity. Cones have a high threshold of sensitivity and good acuity. As vON FRISCH (1925) and WuNDER (1936) point out, the Duplicity Theory is valid for fishes. Though cones in human retina have a sensitivity threshold of 0.03 to $0.1 \mathrm{lux}$, Brunner (1934) has shown that cones in the retina of Phoxinus have a threshold of 0.002 to 0.008 lux.

In view of these works, it is obvious that only the cones are pertinent to an investigation of form perception in fishes.

YAMANOUCHI (1940) measured the density of cones in five regions of the retina of Microcanthus strigatus and obtained the highest density in the temporal region (Table 4).

KaHMANN (1934, 1936) described the fovea centralis in certain fishes and showed that it is most often lacated in the temporal region (Serranus, Julis and Blennius). According to MÜLLER (1952) the various regions of the retina in the guppy (Lebistes reticulatus)

Table 4. Number of cones in $0.1 \mathrm{~mm}$ square in various regions of retina in Microcanthus strigatus

\begin{tabular}{c|c|c|c|c|c} 
Retinal regions & Temporal & Dorsal & Nasal & Ventral & Bottom \\
\hline No. of cones & 155 & 77 & 114 & 97 & 139
\end{tabular}




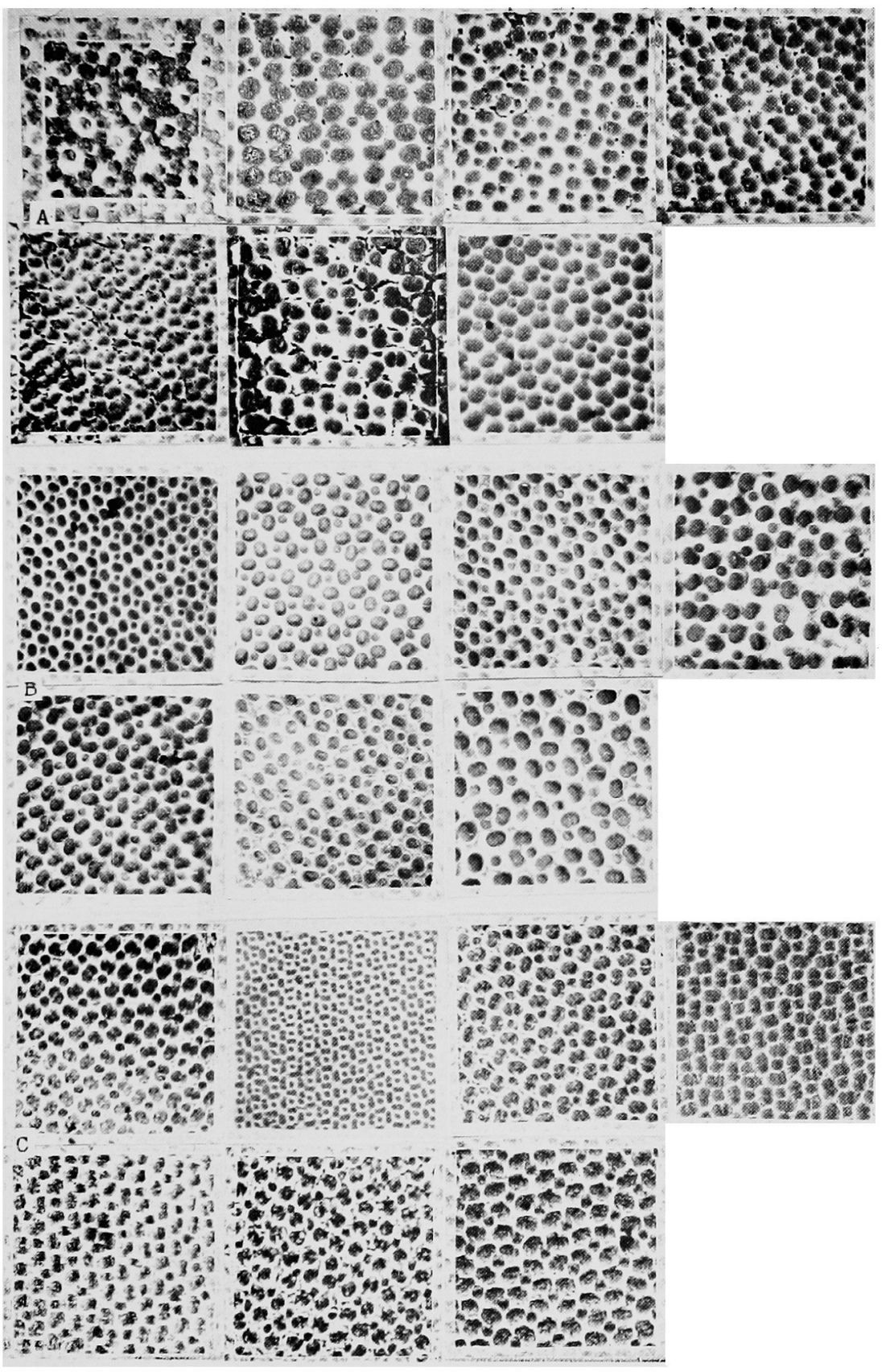

Fig. 3. Microphotograph showing the density of cones. All the squares in the photographs except the upper left one are $0.1 \mathrm{~mm}$ square; the upper left one is $0.03 \mathrm{~mm}$ square,

A. Epinephelus chlorostigma; from left to right, upper row ; temporal, dorso-temporal, ventro-temporal and dorsal, lower row; nasal, ventral and bottom.

B. Lateolabrax japonicus; the same order.

C. Sparus hasta; the same order. 

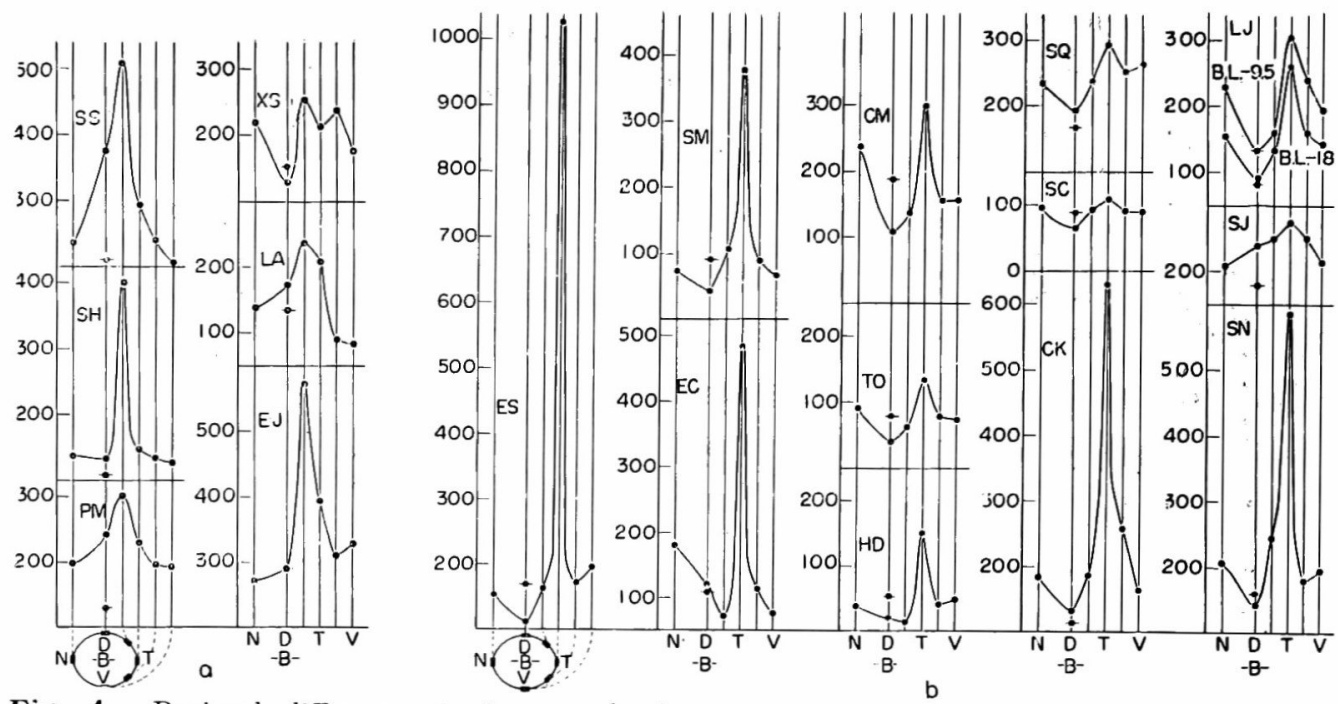

Fig. 4. Regional differences in the cone density.

a. In the fishes which show the maximum density in the dorso-temporal.

b. In the fishes which show the maximum density in the temporal.

SS : Sparus swinhonis, XS: Xesurus scalprum, SH : $S$. hasta, LA : Leiognatus ar. genteus, PM : Pagrosomus major, EJ : Evynnis japo nicus.

ES : Epinephelus seftemfrasciatus, SQ : Seriola quinqueradiata, SM : Sebastiscus marmoratus, SC : Sphaeroides chrysops, EC : Epinephelus chlorostigma, CK : Chelidonichthys kumu, CM : Cantherines modestus, LJ : Lateolabrax japonicus, TO : Therapon oxyrhynchus, SJ : Scomber japonicus, HD : Helicolenus dactylopterus, SN : Sphatroides niphoble's

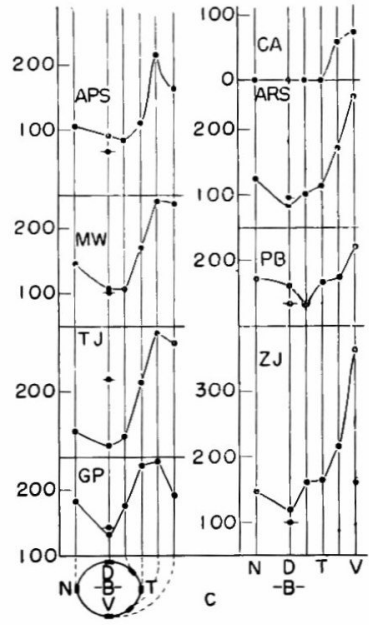

c. In the fishes which show the maximum density in the ventro-temporal or ventral.

APS : Apogon lineatus, CA : Chlorophtalmus albatrossis, MW : Malakichthys wakiyae, ARS: Argentina semifasciata, TJ : Trachurus japonicus, PB : Priacanthus boops, GP : Girella punctata, ZJ : Zenion japonicum,

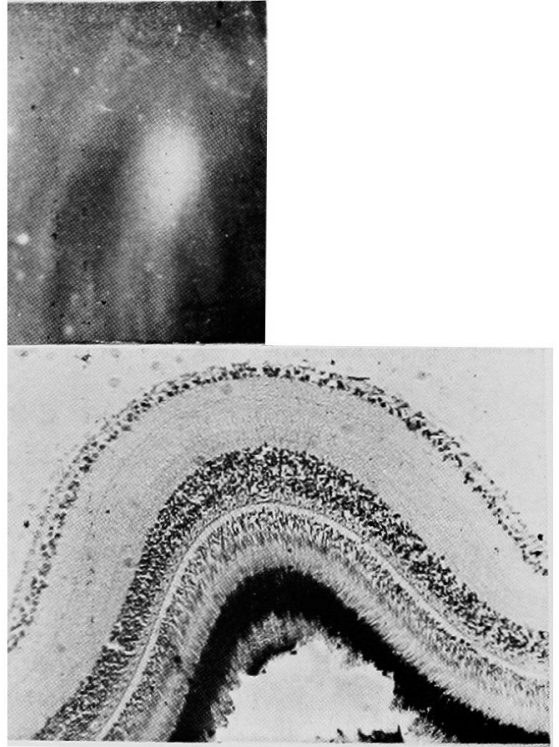

Fig. 5. Area temporalis fixed by Zenker's solution. Upper ; the external appearance of the area in Sebastiscus marmoartus $(\times 30)$.

Lower; the cross section of the area in Epinephelus septemfasciatus $(\times 90)$. 

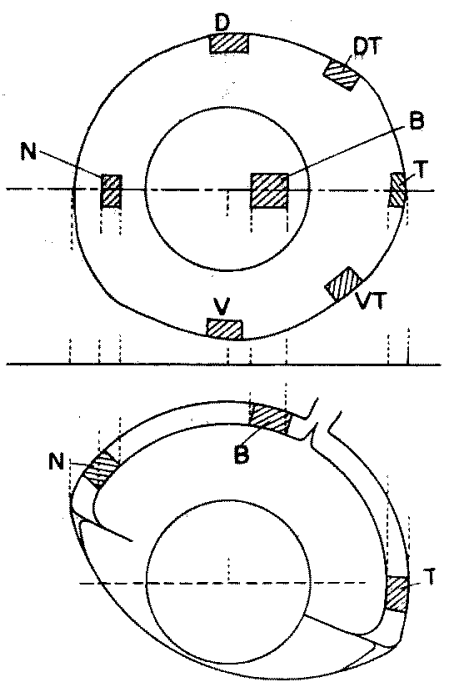

Fig. 2. Schema showing the seven regions in the retina.

T : Temporal, DT : Dorso-temporal, VT : Ventro-temporal, D : Dorsal, N : Nasal, V : Ventral, B : Bottom. can be distinguished by the form and size of cones as well as their density. He further pointed out such regional differences are adapted to the part of visual field that they serve, and remain constant even though new cells are continually being added from the margin of the retina during the developmental stage.

Since cone density, which is an index of resolving power, varies over the retina, the retinas of all the fishes examined in this study were studied topographically.

Method : Eyes were enucleated and fixed in Zenker's solution for 24 hours after removing a small part of the cornea. Each retina was then divided into the following seven regions : temporal, dorso-temporal, ventrotemporal, dorsal, nasal, ventral and bottom (Fig. 2). After routine paraffin imbedding, tangential sections were cut. The cones in $0.1 \mathrm{~mm}$ squares of each region were then counted in photomicrographs (Fig. 3). Since twin and single cones may be present in the teleost retina (Wunder 1936, TAKATSuzi 1939), they were counted separately when they occurred in the species examined.

Fixation and imbedding produced some shrinkage in the retina. According to PATTEN and PHilpotr (1921), the shrinkage was estimated to be $25 \%$ in the pig embryo. Assuming it to be the same in the fish retina, counts were corrected accordingly.

Results : Cone densities by regions are shown in Table 5 and Fig. 4, which are divided into three parts. Part a shows the species that have the maximum density in the dorso-temporal region. Part b shows the species with the greatest density in the temporal region, and part $\mathbf{c}$ shows the species with the greatest density in the ventro-temporal or ventral region.

The focal distances given were calculated from MATthiESSEN's ratio. The "minimum separable angle" was calculated on the assumption that image lines can only be resolved when they fall on cones separated by at least one unstimulated cone. It is

$$
\alpha=\frac{1}{F}\left[\frac{0.1(1+0.25)}{\sqrt{n}} \times 2\right) \text { (Angle in radians) }
$$

where $F$ is the focal distance of the lens, 0.25 is the degree of shrinkage, and $n$ is the maximum number of cones per $0.1 \mathrm{~mm}$ square in one retina and it includes both single and twin cones, although singles and twins, perhaps, play rather different roles in resolution.

The minimum separable angle, which varies from 4.2 minutes in $E$, septemfasciatus to 15.4 minutes in $C$. albatrossis, is obviously more than the resolving power of the lens (Table 3). It may be concluded, therefore, that resolving power is a function of the retina rather than the lens.

When species are compared by the depths at which they live, it is evident that there 
Table 5. The numbers of cones in $0.1 \mathrm{~mm}$ square in various regions of retinae

a. In the fish which have the largest number of cones in the dorso-temporal region

\begin{tabular}{|c|c|c|c|c|c|c|c|c|c|c|}
\hline \multirow[b]{2}{*}{$\begin{array}{c}\text { Species } \\
\text { (Body length in } \mathrm{cm})\end{array}$} & \multirow[b]{2}{*}{$\begin{array}{l}\text { Kinds } \\
\text { of } \\
\text { cones }\end{array}$} & \multicolumn{7}{|c|}{ Retinal regions } & \multirow[b]{2}{*}{$\begin{array}{c}\text { Focal dis- } \\
\text { tance of } \\
\text { lens } \\
(\mathrm{mm})\end{array}$} & \multirow[b]{2}{*}{$\begin{array}{l}\text { Minimum } \\
\text { separable } \\
\text { angle } \\
\text { (minute) }\end{array}$} \\
\hline & & 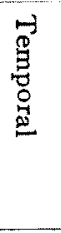 & 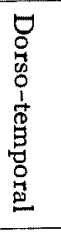 & 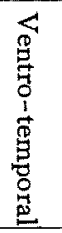 & 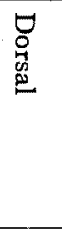 & 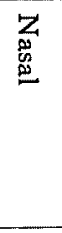 & $\begin{array}{l}\stackrel{4}{0} \\
\stackrel{3}{0} \\
\stackrel{0}{0}\end{array}$ & 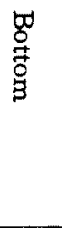 & & \\
\hline $\begin{array}{l}\text { Pagrosomus major } \\
\text { (20) }\end{array}$ & $\begin{array}{l}\text { Twin } \\
\text { Single } \\
\text { Total }\end{array}$ & $\begin{array}{r}151 \\
75 \\
226\end{array}$ & $\begin{array}{l}202 \\
101 \\
303\end{array}$ & $\begin{array}{r}127 \\
68 \\
195\end{array}$ & $\begin{array}{r}160 \\
80 \\
240\end{array}$ & $\begin{array}{r}133 \\
66 \\
199\end{array}$ & $\begin{array}{r}126 \\
65 \\
191\end{array}$ & $\begin{array}{r}88 \\
42 \\
130\end{array}$ & 7.7 & 6.4 \\
\hline $\begin{array}{c}\text { Sparus hasta } \\
\text { (18) }\end{array}$ & $\begin{array}{l}\text { Twin } \\
\text { Single } \\
\text { Total }\end{array}$ & $\begin{array}{r}97 \\
50 \\
147\end{array}$ & $\begin{array}{l}263 \\
137 \\
400\end{array}$ & $\begin{array}{r}90 \\
44 \\
134\end{array}$ & $\begin{array}{r}90 \\
42 \\
132\end{array}$ & $\begin{array}{r}92 \\
46 \\
138\end{array}$ & $\begin{array}{r}84 \\
42 \\
126\end{array}$ & $\begin{array}{r}72 \\
37 \\
109\end{array}$ & 6.9 & 6.2 \\
\hline $\begin{array}{c}\text { Sparus swinhonis } \\
(12)\end{array}$ & $\begin{array}{l}\text { Twin } \\
\text { Single } \\
\text { Total }\end{array}$ & $\begin{array}{r}200 \\
94 \\
294\end{array}$ & $\begin{array}{l}340 \\
169 \\
\mathbf{5 0 9}\end{array}$ & $\begin{array}{r}159 \\
83 \\
242\end{array}$ & $\begin{array}{l}253 \\
126 \\
379\end{array}$ & $\begin{array}{r}157 \\
79 \\
236\end{array}$ & $\begin{array}{r}135 \\
69 \\
204\end{array}$ & $\begin{array}{r}141 \\
69 \\
210\end{array}$ & 5.1 & 6.9 \\
\hline Evynnis japonicus & $\begin{array}{l}\text { Twin } \\
\text { Single } \\
\text { Total }\end{array}$ & $\begin{array}{l}264 \\
129 \\
393\end{array}$ & $\begin{array}{l}380 \\
190 \\
\mathbf{5 7 0}\end{array}$ & $\begin{array}{l}207 \\
103 \\
310\end{array}$ & $\begin{array}{r}193 \\
98 \\
291\end{array}$ & $\begin{array}{r}182 \\
91 \\
273\end{array}$ & $\begin{array}{l}226 \\
113 \\
339\end{array}$ & $\begin{array}{r}195 \\
95 \\
290\end{array}$ & 5.3 & 6.8 \\
\hline $\begin{array}{l}\text { Leiognathus argente- } \\
\text { us } \\
\text { (9.5) }\end{array}$ & $\begin{array}{l}\text { Twin } \\
\text { Single } \\
\text { Total }\end{array}$ & $\begin{array}{r}148 \\
61 \\
209\end{array}$ & $\begin{array}{r}157 \\
79 \\
236\end{array}$ & $\begin{array}{l}57 \\
31 \\
88\end{array}$ & $\begin{array}{r}97 \\
75 \\
172\end{array}$ & $\begin{array}{r}99 \\
41 \\
140\end{array}$ & $\begin{array}{l}56 \\
27 \\
83\end{array}$ & $\begin{array}{r}94 \\
40 \\
134\end{array}$ & 4.8 & 11.6 \\
\hline $\begin{array}{c}\text { Xesurus scalprum } \\
(14)\end{array}$ & $\begin{array}{l}\text { Twin } \\
\text { Single } \\
\text { Total }\end{array}$ & $\begin{array}{r}141 \\
71 \\
212\end{array}$ & $\begin{array}{r}168 \\
84 \\
252\end{array}$ & $\begin{array}{r}156 \\
80 \\
236\end{array}$ & $\begin{array}{r}86 \\
43 \\
129\end{array}$ & $\begin{array}{r}144 \\
74 \\
218\end{array}$ & $\begin{array}{r}115 \\
60 \\
175\end{array}$ & $\begin{array}{r}101 \\
50 \\
151\end{array}$ & 6.4 & 8.5 \\
\hline
\end{tabular}

b. In the fish which have the largest number of cones in the temporal region

\begin{tabular}{|c|c|c|c|c|c|c|c|c|c|c|}
\hline \multirow[b]{2}{*}{$\begin{array}{l}\text { Species } \\
\text { (Body length in } \mathrm{cm} \text { ) }\end{array}$} & \multirow[b]{2}{*}{$\begin{array}{l}\text { Kinds } \\
\text { of } \\
\text { cones }\end{array}$} & \multicolumn{7}{|c|}{ Retinal regions } & \multirow[b]{2}{*}{$\begin{array}{l}\text { Focal dis- } \\
\text { tance of } \\
\text { lens } \\
(\mathrm{mm})\end{array}$} & \multirow[b]{2}{*}{$\begin{array}{l}\text { Minimum } \\
\text { separable } \\
\text { angle } \\
\text { (minute) }\end{array}$} \\
\hline & & 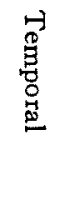 & $\begin{array}{l}\bullet \\
0 \\
0 \\
0 \\
0 \\
1 \\
\overrightarrow{0} \\
3 \\
0 \\
0 \\
0 \\
0\end{array}$ & 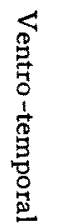 & 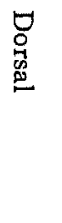 & 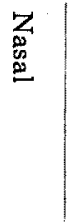 & 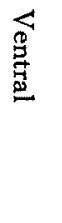 & $\begin{array}{l}\text { gr } \\
\stackrel{0}{0} \\
\stackrel{0}{0} \\
0\end{array}$ & & \\
\hline $\begin{array}{l}\text { Epinephelus septem- } \\
\text { fasciatus } \\
\text { (14) }\end{array}$ & $\begin{array}{l}\text { Twin } \\
\text { Single } \\
\text { Total }\end{array}$ & $\begin{array}{r}700 \\
350 \\
1050\end{array}$ & $\begin{array}{r}132 \\
32 \\
164\end{array}$ & $\begin{array}{r}126 \\
46 \\
172\end{array}$ & $\begin{array}{r}83 \\
27 \\
110\end{array}$ & $\begin{array}{r}176 \\
77 \\
253\end{array}$ & $\begin{array}{r}139 \\
58 \\
197\end{array}$ & $\begin{array}{r}116 \\
54 \\
170\end{array}$ & 6.3 & $4 i^{2}$ \\
\hline $\begin{array}{l}\text { Epinephelus chloro- } \\
\text { stigma }\end{array}$ & $\begin{array}{l}\text { Twin } \\
\text { Single } \\
\text { Total }\end{array}$ & $\begin{array}{l}320 \\
165 \\
\mathbf{4 8 5}\end{array}$ & $\begin{array}{l}55 \\
17 \\
72\end{array}$ & $\begin{array}{r}78 \\
37 \\
115\end{array}$ & $\begin{array}{r}86 \\
37 \\
123\end{array}$ & $\begin{array}{r}125 \\
57 \\
182\end{array}$ & $\begin{array}{l}52 \\
25 \\
77\end{array}$ & $\begin{array}{r}82 \\
29 \\
111\end{array}$ & 6.4 & 6.1 \\
\hline $\begin{array}{l}\text { Sebastiscus marmo- } \\
\text { ratus }\end{array}$ & $\begin{array}{l}\text { Twin } \\
\text { Single } \\
\text { Total }\end{array}$ & $\begin{array}{r}300 \\
80 \\
380\end{array}$ & $\begin{array}{r}73 \\
32 \\
105\end{array}$ & $\begin{array}{l}60 \\
30 \\
90\end{array}$ & $\begin{array}{r}34 \\
8 \\
42\end{array}$ & $\begin{array}{l}59 \\
15 \\
74\end{array}$ & $\begin{array}{l}58 \\
11 \\
69\end{array}$ & $\begin{array}{l}60 \\
30 \\
90\end{array}$ & 6.6 & 6.7 \\
\hline $\begin{array}{l}\text { Helicolenus dact ylo- } \\
\text { pterus } \\
\qquad \text { (17) }\end{array}$ & $\begin{array}{l}\text { Twin } \\
\text { Single } \\
\text { Total }\end{array}$ & $\begin{array}{r}150 \\
0 \\
150\end{array}$ & $\begin{array}{r}16 \\
0 \\
16\end{array}$ & $\begin{array}{r}43 \\
0 \\
43\end{array}$ & $\begin{array}{r}20 \\
0 \\
20\end{array}$ & $\begin{array}{r}39 \\
0 \\
39\end{array}$ & $\begin{array}{r}49 \\
0 \\
49\end{array}$ & $\begin{array}{r}30 \\
0 \\
30\end{array}$ & 6.6 & 10.6 \\
\hline $\begin{array}{l}\text { Therapon oxyrhync- } \\
\text { hus } \\
\text { (20) }\end{array}$ & $\begin{array}{l}\text { Twin } \\
\text { Single } \\
\text { Total }\end{array}$ & $\begin{array}{r}88 \\
45 \\
133\end{array}$ & $\begin{array}{l}42 \\
19 \\
61\end{array}$ & $\begin{array}{l}55 \\
22 \\
77\end{array}$ & $\begin{array}{l}30 \\
10 \\
40\end{array}$ & $\begin{array}{l}65 \\
28 \\
93\end{array}$ & $\begin{array}{l}52 \\
22 \\
74\end{array}$ & $\begin{array}{l}53 \\
26 \\
79\end{array}$ & 7.7 & 9.7 \\
\hline $\begin{array}{c}\text { Cantherines modestus } \\
(28)\end{array}$ & $\begin{array}{l}\text { Twin } \\
\text { Single } \\
\text { Total }\end{array}$ & $\begin{array}{l}197 \\
102 \\
299\end{array}$ & $\begin{array}{r}87 \\
50 \\
137\end{array}$ & $\begin{array}{r}104 \\
52 \\
156\end{array}$ & $\begin{array}{r}74 \\
34 \\
108\end{array}$ & $\begin{array}{r}161 \\
78 \\
239\end{array}$ & $\begin{array}{r}104 \\
53 \\
157\end{array}$ & $\begin{array}{r}125 \\
64 \\
189\end{array}$ & 7.7 & $\begin{array}{l}6.4 \\
.\end{array}$ \\
\hline
\end{tabular}




\begin{tabular}{|c|c|c|c|c|c|c|c|c|c|c|}
\hline $\begin{array}{l}\text { Chelidonichthys } \\
\text { kumu }\end{array}$ & $\begin{array}{l}\text { Twin } \\
\text { Single } \\
\text { Total }\end{array}$ & $\begin{array}{l}420 \\
210 \\
630\end{array}$ & $\begin{array}{r}124 \\
62 \\
186\end{array}$ & $\begin{array}{r}172 \\
87 \\
259\end{array}$ & $\begin{array}{r}85 \\
46 \\
131\end{array}$ & $\begin{array}{r}125 \\
60 \\
185\end{array}$ & $\begin{array}{r}105 \\
59 \\
164\end{array}$ & $\begin{array}{r}78 \\
37 \\
115\end{array}$ & 5.0 & 6.8 \\
\hline $\begin{array}{l}\text { Sphaeroides niphob- } \\
\text { les }\end{array}$ & $\begin{array}{l}\text { Twin } \\
\text { Single } \\
\text { Total }\end{array}$ & $\begin{array}{l}390 \\
195 \\
\mathbf{5 8 5}\end{array}$ & $\begin{array}{r}160 \\
83 \\
243\end{array}$ & $\begin{array}{r}119 \\
60 \\
179\end{array}$ & $\begin{array}{r}96 \\
47 \\
143\end{array}$ & $\begin{array}{r}138 \\
69 \\
207\end{array}$ & $\begin{array}{r}129 \\
67 \\
196\end{array}$ & $\begin{array}{r}108 \\
52 \\
160\end{array}$ & 5.1 & 7.0 \\
\hline $\begin{array}{c}\text { Sphaeroides chrysops } \\
\text { (17) }\end{array}$ & $\begin{array}{l}\text { Twin } \\
\text { Single } \\
\text { Total }\end{array}$ & $\begin{array}{r}72 \\
35 \\
107\end{array}$ & $\begin{array}{l}60 \\
31 \\
91\end{array}$ & $\begin{array}{l}60 \\
30 \\
90\end{array}$ & $\begin{array}{l}42 \\
22 \\
64\end{array}$ & $\begin{array}{l}65 \\
31 \\
96\end{array}$ & $\begin{array}{l}56 \\
32 \\
88\end{array}$ & $\begin{array}{l}61 \\
28 \\
89\end{array}$ & 5.8 & 14.3 \\
\hline $\begin{array}{l}\text { Seriola quinquerad- } \\
\text { iata } \\
\end{array}$ & $\begin{array}{l}\text { Twin } \\
\text { Single } \\
\text { Total }\end{array}$ & $\begin{array}{r}195 \\
97 \\
292\end{array}$ & $\begin{array}{r}156 \\
81 \\
237\end{array}$ & $\begin{array}{r}164 \\
87 \\
251\end{array}$ & $\begin{array}{r}127 \\
66 \\
193\end{array}$ & $\begin{array}{r}154 \\
79 \\
233\end{array}$ & $\begin{array}{r}175 \\
89 \\
264\end{array}$ & $\begin{array}{r}111 \\
57 \\
168\end{array}$ & 5.8 & 8.7 \\
\hline $\begin{array}{l}\text { Lateolabrax japon- } \\
\text { icus } \\
\text { (18) }\end{array}$ & $\begin{array}{l}\text { Twin } \\
\text { Single } \\
\text { Total }\end{array}$ & $\begin{array}{r}181 \\
78 \\
259\end{array}$ & $\begin{array}{r}89 \\
43 \\
132\end{array}$ & $\begin{array}{r}106 \\
52 \\
158\end{array}$ & $\begin{array}{l}62 \\
28 \\
90\end{array}$ & $\begin{array}{r}104 \\
50 \\
154\end{array}$ & $\begin{array}{r}98 \\
44 \\
142\end{array}$ & $\begin{array}{l}55 \\
27 \\
82\end{array}$ & 6.3 & 8.5 \\
\hline$(9.5)$ & $\begin{array}{l}\text { Twin } \\
\text { Single } \\
\text { Total }\end{array}$ & $\begin{array}{l}202 \\
100 \\
\mathbf{3 0 2}\end{array}$ & $\begin{array}{r}105 \\
53 \\
158\end{array}$ & $\begin{array}{r}161 \\
80 \\
241\end{array}$ & $\begin{array}{r}89 \\
44 \\
133\end{array}$ & $\begin{array}{r}153 \\
77 \\
230\end{array}$ & $\begin{array}{r}128 \\
65 \\
193\end{array}$ & $\begin{array}{r}88 \\
45 \\
133\end{array}$ & 4.2 & 11.7 \\
\hline $\begin{array}{c}\text { Scomber japonicus } \\
(17)\end{array}$ & $\begin{array}{l}\text { Twin } \\
\text { Single } \\
\text { Total }\end{array}$ & $\begin{array}{r}178 \\
96 \\
274\end{array}$ & $\begin{array}{l}125 \\
124 \\
249\end{array}$ & $\begin{array}{r}159 \\
91 \\
250\end{array}$ & $\begin{array}{l}130 \\
106 \\
236\end{array}$ & $\begin{array}{r}154 \\
55 \\
209\end{array}$ & $\begin{array}{r}146 \\
68 \\
214\end{array}$ & $\begin{array}{r}120 \\
58 \\
178\end{array}$ & 6.9 & 7.5 \\
\hline
\end{tabular}

Table 5 .

c. In the fish which have the largest number of cones in the ventro-temporal or ventral region

\begin{tabular}{|c|c|c|c|c|c|c|c|c|c|c|}
\hline \multirow[b]{2}{*}{$\begin{array}{c}\text { Species } \\
\text { (Body length in } \mathrm{cm} \text { ) }\end{array}$} & \multirow[b]{2}{*}{$\begin{array}{l}\text { Kinds } \\
\text { of } \\
\text { cones }\end{array}$} & \multicolumn{7}{|c|}{ Retinal regions } & \multirow[b]{2}{*}{$\begin{array}{c}\text { Focal dis- } \\
\text { tance of } \\
\text { lens } \\
(\mathrm{mm})\end{array}$} & \multirow[b]{2}{*}{$\begin{array}{l}\text { Minimum } \\
\text { separable } \\
\text { angle } \\
\text { (minute) }\end{array}$} \\
\hline & & 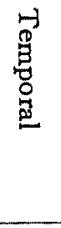 & $\begin{array}{l}\forall \\
0 \\
0 \\
0 \\
1 \\
0 \\
3 \\
8 \\
8 \\
0 \\
0\end{array}$ & 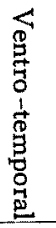 & $\begin{array}{l}\forall \\
\stackrel{0}{0} \\
2\end{array}$ & 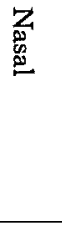 & 总 & $\begin{array}{l}\infty \\
\stackrel{0}{0} \\
\frac{0}{3}\end{array}$ & & \\
\hline$\underset{(30)}{\text { Girella }} \underset{\text { punciaia }}{ }$ & $\begin{array}{l}\text { Twin } \\
\text { Single } \\
\text { Total }\end{array}$ & $\begin{array}{r}151 \\
86 \\
237\end{array}$ & $\begin{array}{r}114 \\
61 \\
175\end{array}$ & $\begin{array}{r}162 \\
82 \\
244\end{array}$ & $\begin{array}{r}83 \\
49 \\
132\end{array}$ & $\begin{array}{r}120 \\
63 \\
183\end{array}$ & $\begin{array}{r}126 \\
65 \\
191\end{array}$ & $\begin{array}{r}96 \\
47 \\
143\end{array}$ & 7.1 & 7.8 \\
\hline$\underset{\text { (13) }}{\text { Trachurus japonicus }}$ & $\begin{array}{l}\text { Twin } \\
\text { Single } \\
\text { Total }\end{array}$ & $\begin{array}{r}141 \\
71 \\
212\end{array}$ & $\begin{array}{r}87 \\
43 \\
130\end{array}$ & $\begin{array}{r}190 \\
98 \\
288\end{array}$ & $\begin{array}{r}77 \\
40 \\
117\end{array}$ & $\begin{array}{r}92 \\
46 \\
138\end{array}$ & $\begin{array}{r}182 \\
90 \\
272\end{array}$ & $\begin{array}{r}145 \\
74 \\
219\end{array}$ & 5.9 & 8.6 \\
\hline $\begin{array}{l}\text { Malakichthys waki- } \\
\text { yae (9) }\end{array}$ & $\begin{array}{l}\text { Twin } \\
\text { Single } \\
\text { Total }\end{array}$ & $\begin{array}{r}166 \\
0 \\
166\end{array}$ & $\begin{array}{r}107 \\
0 \\
107\end{array}$ & $\begin{array}{r}242 \\
0 \\
242\end{array}$ & $\begin{array}{r}108 \\
0 \\
108\end{array}$ & $\begin{array}{r}145 \\
0 \\
145\end{array}$ & $\begin{array}{r}237 \\
0 \\
237\end{array}$ & $\begin{array}{r}105 \\
0 \\
105\end{array}$ & 7.0 & 7.9 \\
\hline $\begin{array}{c}\text { Apogon lineatus } \\
(8)\end{array}$ & $\begin{array}{l}\text { Twin } \\
\text { Single } \\
\text { Total }\end{array}$ & $\begin{array}{r}96 \\
13 \\
109\end{array}$ & $\begin{array}{r}76 \\
8 \\
84\end{array}$ & $\begin{array}{r}200 \\
15 \\
215\end{array}$ & $\begin{array}{l}79 \\
13 \\
92\end{array}$ & $\begin{array}{r}96 \\
12 \\
108\end{array}$ & $\begin{array}{r}153 \\
10 \\
163\end{array}$ & $\begin{array}{l}56 \\
11 \\
67\end{array}$ & 4.1 & 14.3 \\
\hline Zenion japonicum & $\begin{array}{l}\text { Twin } \\
\text { Single } \\
\text { Total }\end{array}$ & $\begin{array}{r}163 \\
0 \\
163\end{array}$ & $\begin{array}{r}161 \\
0 \\
161\end{array}$ & $\begin{array}{r}215 \\
0 \\
215\end{array}$ & $\begin{array}{r}119 \\
0 \\
119\end{array}$ & $\begin{array}{r}148 \\
0 \\
148\end{array}$ & $\begin{array}{r}361 \\
0 \\
361\end{array}$ & $\begin{array}{r}100 \\
0 \\
100\end{array}$ & 7.0 & 6.5 \\
\hline $\begin{array}{c}\text { Priacanthus boops } \\
\text { (15) }\end{array}$ & $\begin{array}{l}\text { Twin } \\
\text { Single } \\
\text { Total }\end{array}$ & $\begin{array}{r}112 \\
53 \\
165\end{array}$ & $\begin{array}{r}96 \\
41 \\
137\end{array}$ & $\begin{array}{r}104 \\
68 \\
172\end{array}$ & $\begin{array}{r}118 \\
44 \\
162\end{array}$ & $\begin{array}{r}118 \\
53 \\
171\end{array}$ & $\begin{array}{r}170 \\
50 \\
220\end{array}$ & $\begin{array}{r}94 \\
39 \\
133\end{array}$ & 10.0 & 5.8 \\
\hline $\begin{array}{l}\text { Argentina semifas- } \\
\text { ciata }\end{array}$ & $\begin{array}{l}\text { Twin } \\
\text { Single } \\
\text { Total }\end{array}$ & $\begin{array}{r}114 \\
0 \\
114\end{array}$ & $\begin{array}{r}103 \\
0 \\
103\end{array}$ & $\begin{array}{r}170 \\
0 \\
170\end{array}$ & $\begin{array}{r}84 \\
0 \\
84\end{array}$ & $\begin{array}{r}123 \\
0 \\
123\end{array}$ & $\begin{array}{r}251 \\
0 \\
251\end{array}$ & $\begin{array}{r}93 \\
0 \\
93\end{array}$ & 5.9 & 9.2 \\
\hline $\begin{array}{l}\text { Chlorophthalmus } \\
\text { albatrossis } \\
\text { (13) }\end{array}$ & $\begin{array}{l}\text { Twin } \\
\text { Single } \\
\text { Total }\end{array}$ & $\begin{array}{l}0 \\
0 \\
0\end{array}$ & $\begin{array}{l}0 \\
0 \\
0\end{array}$ & $\begin{array}{r}58 \\
0 \\
58\end{array}$ & $\begin{array}{l}0 \\
0 \\
0\end{array}$ & $\begin{array}{l}0 \\
0 \\
0\end{array}$ & $\begin{array}{r}72 \\
0 \\
72\end{array}$ & $\begin{array}{l}0 \\
0 \\
0\end{array}$ & 6.6 & 15.4 \\
\hline
\end{tabular}

is a relationship between depth and the relative abundance of twin and single cones. Scomber, which lives very close to the surface, has almost equal numbers of twin and 
single cones in the dorso-temporal region of retina. Most of the other species examined live rather near the surface and have half as many single as twin cones. However, Helicolenus, Malakichthys, Zenion, Argentina and Chlorophthalmus, which were caught at depths of 100 to 300 meters, did not have single cones, and did have well developed tapeta lucida. Chlorophthalmus, furthermore, had twin cones only in the ventral and ventrotemporal regions. It usually inhabits depths greater than 300 meters.

The above relationship indicates that twin cones are more sensitive to light than single cones, and there are definite physiological and ecological differences between them. It has also been shown (Table 5 and Fig. 4) that cone density varies by region over the retina. The relationship between the different regions and the portions of the optical field they subtend will be discussed under Visual axis.

Visual acuity depends on the size of the retinal image and the cone density. The greater the number of cones over which the image is spread, the better the resolution. If cone density is constant throughout life, resolving power would increase as the lens grows. However, from MülLER's work on the guppy and the present data on Lateolabrax (Table $5 \mathrm{~b}$ and Fig. $4 \mathrm{~b}$ ), cone density is higher in the younger stages. Consequently, lens size is at least partly compensated for by cone density.

Nothing resembling the fovea centralis or lateralis described by KAHMANN $(1934,1936)$ was found in the species listed in Table 5. However, in the bright adapted retinas of certain Japanese Serranids (E. septemfasciatus, E. chlorostigma) and Scorpaenids ( $S$. marmoratus and $H$. dactylopterus) a small elevation possibly representing an area temporalis was found (Fig. 5). These specimens were fixed in Zenker's solution, as were all other preparations. Both twin and single cones occurred in this area. But since no such elevation could be found in either fresh or dark adapted eyes fixed in Zenker's solution, and because the pigment layer was separated from the chorioid layer at this point (Fig. 5), it is strongly suspected to be an artifact.

Thus most fish have an area at least somewhat specialized for more acute vision, even though true areae and foveae may be absent. Although the extent of this specialization may be judged from Fig. 4, a more comparable index is the ratio of cone density in the highest region to the average cone density of the rest of the retina (Table 6). Table 6 shows that five demersal and somewhat sedentary species (Chlorophthalmus, two species of Epinephelus, Sebastiscus and Helicolenus) have the highest degree of specialization, and that two of them, Chlorophthalmus and Helicolenus have relatively great regional specialization in spite of the fact that they live at the depths greater than 100 meters where light is scarce. It should also be noted that these two have large separable angles. The lower degree of specialization in Scomber and Seriola can almost certainly be attributed to the fact that they are pelagic and therefore probably find good periscopy advantageous. No explanation can be given for the rather striking difference between $S$. niphobles and $S$. chrysops, because very little is known of their behaviour

According to BrunNer (1934) and YamanouchI (1940), the least separable visual angle in Phoxinus and Microcanthus approximates the angle subtended by one cone, suggesting that there are separate conductive paths from each cone to the higher centers in the 
Table 6. The degree of specialization of the area

\begin{tabular}{|c|c|c|}
\hline Species & Degree of specialization & $\begin{array}{l}\text { Minimum separable angle } \\
\text { (minute) }\end{array}$ \\
\hline $\begin{array}{l}\text { Scomber japonicus } \\
\text { Sphaeroides chrysops } \\
\text { Seriola quinqueradiata } \\
\text { Xesurus scalprum }\end{array}$ & $\begin{array}{l}1.21 \\
1.24 \\
1.25 \\
1.35\end{array}$ & $\begin{array}{r}7.5 \\
14.3 \\
8.7 \\
8.5\end{array}$ \\
\hline $\begin{array}{l}\text { Girella punctata } \\
\text { Priacanthus boops } \\
\text { Pagrosomus major } \\
\text { Trachurus japonicus }\end{array}$ & $\begin{array}{l}1.38 \\
1.40 \\
1.54 \\
1.59\end{array}$ & $\begin{array}{l}7.8 \\
5.8 \\
6.4 \\
8.6\end{array}$ \\
\hline \multirow{2}{*}{$\begin{array}{l}\text { Lateolabrax japonicus } \\
\text { Body length } 9.5 \mathrm{~cm} \\
\text { Malakichthys wakiyae } \\
\text { Evynnis japonicus } \\
\text { Leiognathus argenteus }\end{array}$} & 1.66 & 11.7 \\
\hline & $\begin{array}{l}1.67 \\
1.70 \\
1.72\end{array}$ & $\begin{array}{r}7.9 \\
6.8 \\
11.6\end{array}$ \\
\hline \multirow{2}{*}{$\begin{array}{l}\text { Cantherines modestus } \\
\text { Therapon ox yrhynchus } \\
\text { Sparus swinhonis } \\
\text { Lateolabrax japonicus } \\
\text { Body length } 17 \mathrm{~cm}\end{array}$} & $\begin{array}{l}1.82 \\
1.88 \\
1.95\end{array}$ & $\begin{array}{l}6.4 \\
9.7 \\
6.9\end{array}$ \\
\hline & 2.05 & 8.7 \\
\hline $\begin{array}{l}\text { Apogon lineatus } \\
\text { Argentina semifasciata } \\
\text { Zenion japonicum } \\
\text { Sparus hasta }\end{array}$ & $\begin{array}{l}2.07 \\
2.20 \\
2.39 \\
3.05\end{array}$ & $\begin{array}{r}14.3 \\
9.2 \\
6.5 \\
6.2\end{array}$ \\
\hline $\begin{array}{l}\text { Sphaeroides niphobles } \\
\text { Chelidonichthys kumu } \\
\text { Epinephelus chlorostigma } \\
\text { Helicolenus dactylopterus }\end{array}$ & $\begin{array}{l}3.11 \\
3.63 \\
4.19 \\
4.58\end{array}$ & $\begin{array}{r}7.0 \\
6.8 \\
6.1 \\
10.6\end{array}$ \\
\hline $\begin{array}{l}\text { Sebastiscus marmoratus } \\
\text { Epinephelus septemfasciatus } \\
\text { Chlorophthalmus albatrossis }\end{array}$ & $\begin{array}{l}4.86 \\
6.52 \\
7.46\end{array}$ & $\begin{array}{r}6.7 \\
4.2 \\
15.4\end{array}$ \\
\hline
\end{tabular}

brain as is the case in primates. This can only be ascertained by further neuroanatomical investigation.

\section{Accommodation}

BEER (1894), in his thorough investigation of accommodation in the fish eye, showed that fish eyes are myopic in the resting state, and that accommodation to distant objects is accomplished by retraction of the lens towards the retina. Retraction is effected by the musculus retractor lentis (Campanula Halleri). BEER's views have been confirmed by the work of HESS (1912), MEADER (1936), and YAMAMoto (1931).

In the present study, accommodation has been measured in 13 marine teleosts. The method employed differed somewhat from BEER's, and has produced additional information.

Method : Fishes, generally speaking, have monocular visual fields of approximately $180^{\circ}$, due in part to the lateral location of the eyes and in part to the spherical lens that produces beyond the pupil. Anteriorly, the monocular fields overlap to a greater or lesser extent, forming a binocular field. The functional importance of the binocular field in fish has been demonstrated by HERTER's experiments on binocular vision (1930).

In the present study, refraction was measured from six points in the visual field : fore, upper-fore, lower-fore, upper, lower, and lateral (Fig. 6). In most cases, the fore and upper-fore or lower-fore fell within the binocular field, a further indication of 


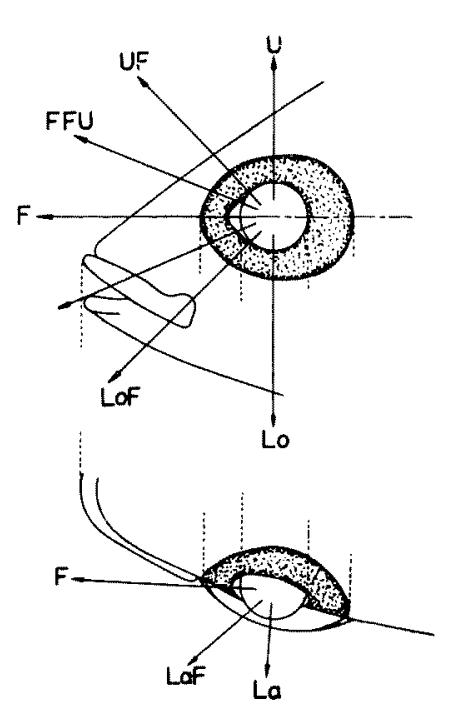

Fig. 6. Labeling of visual directions.

U : Upper, UF : Upper-fore, FFU

: Fore-fore-Upper, F : Fore, LoF :

Lower-fore, Lo : Lower, La : Later-

al, LaF : Lateral-fore.

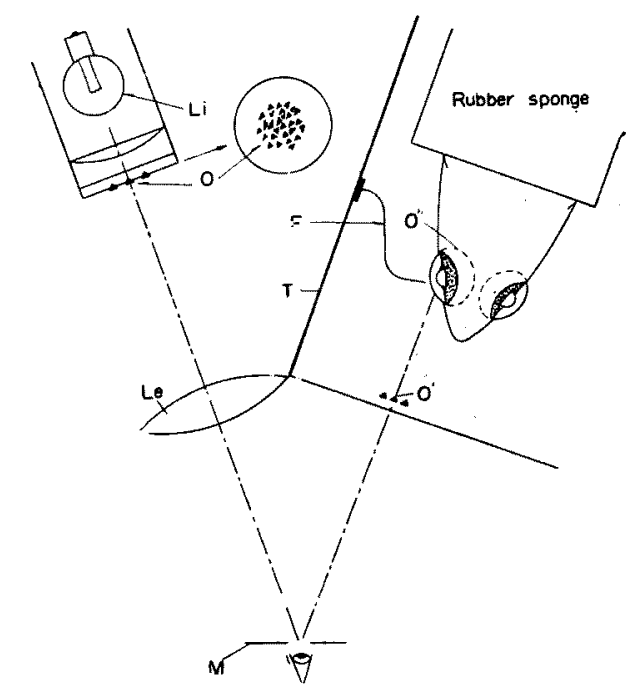

Fig. 7. Schema showing the method of measuring the refraction of fish eye. Explanation in the text.

the functional importance of this zone in view of the fact that these portions of the visual field are served by the temporal regions of the retina, where cone density is highest.

Refraction was measured with an instrument similar to the refractometer used for human eyes. In Fig. 7, which illustrates the refractometer used, 0 is a pattern of black triangles of about 2 millimeters mounted in front of the lamp. Le is a convex lens with a focal distance of about $35 \mathrm{~cm}$. $M$ is a mirror with a small hole in the center, and $T$ is a tank of sea water with one glass wall. The specimen under examination was fixed rigidly in the tank between two pieces of rubber sponge.

Measurement were made in the dark in the following manner. The pattern 0 was reflected into the eye and brought to maximum focus there as viewed by the observer through the hole in the mirror. This was accomplished by moving the lamp with the pattern mounted on it. Then, $0^{\prime}$ is the real image of 0 , formed by the lens (Le) and reflected by the mirror $(M)$. The plane of $0^{\prime}$ was ascertained by a movable white screen placed in the tank, and the distance between it and the center of the lens of the fish was measured. The method of the measurement was as follows : after the maximum focus was. brought and the supposed position of the center of the lens was pointed by the tip of the flexible fuse $(F)$, the fish was removed, and then the position of $0^{\prime}$ was ascertained by the white screen. The distance from the tip of the fuse to the screen was measured along the light direction by a pair of dividers or a rule.

When $0^{\prime}$ was in front of the eye, as in Fig. 7, the eye was myopic, when behind, the eye was hypermetropic. When $0^{\prime}$ was very far either in front of or behind the fish eye, it was emmetropic or nearly so, but it was not possible to see $0^{\prime \prime}$ under this condition. Consequently, an auxiliary concave lens was placed in front of the eye for the measurement, and its effect later subtracted. 


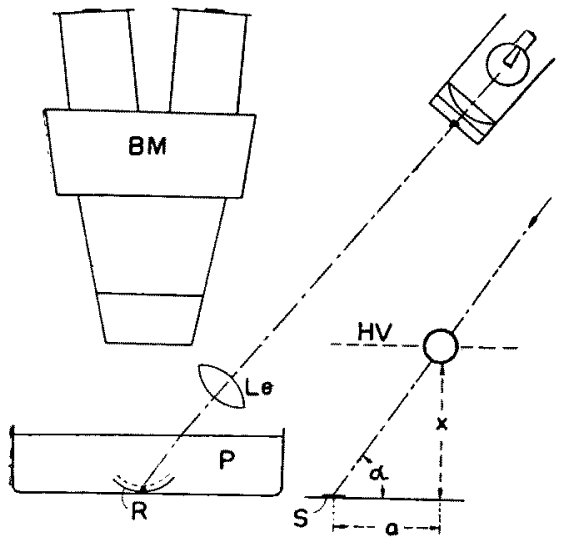

b

The assumption is made, in this method, that the image $0^{\prime \prime}$ is focused on the photosensitive layer of the retina. If $0^{\prime \prime}$ was focused some other level of the retina, considerable corrections would be necessary in view of the relative thickness of the fish retina and the short focal distance of the lens. The validity of this assumption was determined in the following way. A small piece of living retina was excised from Carassius auratus and placed in physiological salt solution.

The triangular pattern was focused on it, as shown in Fig. 8, then examined under a binocular microscope. The image was obviously formed beyond the inner limiting membrane, which is readily identified by the presence of hyaloid or vitreal vessels distributed over its surface.

Fig. 8. a. R : Piece of retina, $\mathrm{P}$ : Phy siological salt solution, Le : Convex lens (focal distance $24 \mathrm{~mm}$ ), BM : Binocularmicroscope.

b. HV : Hyaloid vessel, $\mathrm{S}$ : Shadow of $\mathrm{HV}$.

The distance, $\mathrm{x}$, between the hyaloid vessels and the plane of focus was determined by simple triangulation (Fig. $8 \mathrm{~b}$ ),

$$
\mathrm{x}=a \times \tan \alpha=0.19 \times \tan 38^{\circ}=0.15 \mathrm{~mm}
$$

where $\mathrm{a}$ is the horizontal distance between the hyaloid vessel and its shadow on the plane of focus, and $\tan \alpha$ is the incident angle of illumination source. The piece of retina was then fixed in Zenker's solution and sectioned. Direct measurement of the sectioned material showed the inner limiting membrane and the photosensitive layer of the retina to be $0.13 \mathrm{~mm}$ apart. Correction for shrinkage shows that this measurement agrees almost perfectly with the $0.15 \mathrm{~mm}$ obtained by triangulation.

$$
0.13 \times(1+0.25)=0.16 \mathrm{~mm}
$$

Thus it is safe to assume the image $0^{\prime \prime}$ in Fig. 7 is formed on the photosensitive layer of the retina.

At least two sets of refraction measurements were obtained for each species : one without treatment (pre-treatment) the other one or more of the five treatments (post-treatment). These were (1) severing the optic nerve from inside the mouth, (2) killing by laceration of the heart, (3) intramuscular injection of $0.5 \mathrm{cc}$ of $1 \%$ atropine solution, (4) injection of $1 \mathrm{cc}$ of Amelizol, containing $3 \mathrm{mg}$ of d-tubocurarine chloride and $5 \mathrm{mg}$ of chlorobutanol, (5) injection of $0.5 \mathrm{cc}$ of a $1 \%$ aqueous solution of curare (d-tubocurarine chloride). The last two were included in order to test BEER's data, since he used curare as the anaesthetic. Severing the optic nerve was effective immediately, while the injections were not effective for 10 or 15 minutes.

Results : Refraction values for thirteen species are given in diopter units in Table 7 $\mathrm{a}, \mathrm{b}, \mathrm{c}$. Hypermetropia is indicated by + , myopia by - , and emmetropia by 0 . Error of measurement may be about \pm 1 diopter.

Table 7 shows that all eyes were hypermetropic or emmetropic in pre-treatment, only becoming myopic in certain directions after treatment. Incidentally, no differences were 
Table 7. The values of the refraction (Diopter)

a. In the fishes which show the highest myopia in the lower-fore direction

\begin{tabular}{|c|c|c|c|c|c|c|c|c|c|c|c|c|c|}
\hline & & & Pre-trea & atment & & & & & Cost-tre & eatment & & & \\
\hline $\begin{array}{l}\text { Species } \\
\text { (Body } \\
\text { length cm) }\end{array}$ & $\frac{7}{3}$ & $\begin{array}{l}5 \\
\stackrel{5}{D} \\
\stackrel{5}{6}\end{array}$ & 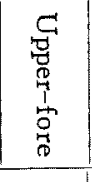 & 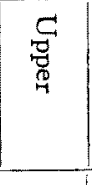 & $\begin{array}{l}5 \\
0 \\
0 \\
9 \\
1 \\
0 \\
0 \\
0\end{array}$ & 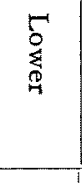 & $\begin{array}{l}3 \\
\text { के } \\
\text { है }\end{array}$ & 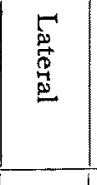 & 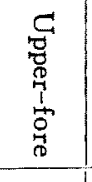 & 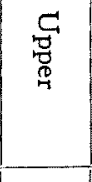 & \begin{tabular}{l}
5 \\
0 \\
\multirow{4}{*}{} \\
9 \\
1 \\
1 \\
0 \\
0 \\
0
\end{tabular} & 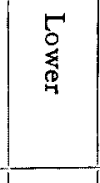 & 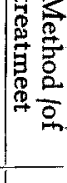 \\
\hline $\begin{array}{l}\text { Pagrosomus } \\
\text { major (18) }\end{array}$ & +1.7 & & & +5.0 & +1.3 & 0 & -5.9 & +2.0 & +4.0 & & -11 & & At \\
\hline$\prime \prime \quad(7)$ & +7.7 & +7.1 & +10 & & & & -15 & +10 & +2.5 & +8.3 & -22 & +6.7 & At \\
\hline Mean & +4.7 & +4.8 & +6.0 & +5.0 & +1.3 & 0 & $-10.5 \mid$ & +6.0 & +6.3 & +5.2 & -16.5 & +0.2 & \\
\hline $\begin{array}{r}\text { parus hasta } \\
(16.5)\end{array}$ & +1.3 & 0 & +2.0 & +2.1 & +2.9 & +3.2 & -7.7 & +1.3 & -5.0 & & & & $\mathrm{~S}$ \\
\hline " (16) & 0 & +1.3 & +1.8 & +1.8 & +0.9 & +0.9 & -13 & +4.2 & +2.0 & +17 & -13 & -6.7 & $\mathrm{~s}$ \\
\hline "I (16) & +1.4 & & & & & & -13 & +3.1 & & +9.1 & -13 & -10 & $\mathrm{~K}$ \\
\hline Mean & +0.9 & +0.7 & +1.9 & +2.0 & +1.9 & +2.1 & -11.2 & +2.9 & -1.5 & +12.6 & -13 & -8.9 & 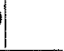 \\
\hline $\begin{array}{l}\text { Evynnis } \\
\text { japonicus (10) }\end{array}$ & +5.3 & +3.9 & +4.8 & +5.3 & +4.8 & +4.3 & -8.3 & +6.7 & +1.7 & +7.7 & -14 & +3.1 & $S$ \\
\hline "(10) $\begin{array}{l}\text { L. eye } \\
\text { R. eye }\end{array}$ & $\begin{array}{l}+4.3 \\
+3.0\end{array}$ & $\begin{array}{l}+6.3 \\
+4.0\end{array}$ & $\begin{array}{l}+6.3 \\
+5.6\end{array}$ & $\begin{array}{l}+7.2 \\
+5.9\end{array}$ & $\begin{array}{l}+3.9 \\
+5.0\end{array}$ & $\begin{array}{l}+3.7 \\
+5.3\end{array}$ & $\begin{array}{l}-13 \\
-13\end{array}$ & $\begin{array}{l}+5.9 \\
+5.9\end{array}$ & $\begin{array}{l}-5.6 \\
-4.3\end{array}$ & $\begin{array}{l}+7.2 \\
+7.7\end{array}$ & $\begin{array}{l}-13 \\
-14\end{array}$ & $\begin{array}{l}+2.9 \\
-5.6\end{array}$ & $S$ \\
\hline$"(10)$ & +2.2 & +2.5 & +2.5 & +2.5 & +0.8 & 0 & -13 & +2.2 & -7.7 & +5.9 & -11 & +1.6 & S \\
\hline Mean & +3.7 & +4.2 & +4.8 & +5.2 & +3.6 & +3.3 & -11.8 & +5.2 & -4.0 & +7.1 & -13 & +0.5 & \\
\hline $\begin{array}{l}\text { Leiognathus } \\
\text { argenteus (9.5) }\end{array}$ & +4.2 & +6.7 & +5.0 & +10 & +2.9 & +2.1 & -14 & +10 & -5.0 & +8.3 & -14 & -14 & $\mathrm{~S}$ \\
\hline $\begin{array}{l}\text { Xesurus L.eye } \\
\text { scalprum R.eye } \\
\text { (14) }\end{array}$ & $\begin{array}{l}+3.9 \\
+4.0\end{array}$ & +3.9 & +2.0 & +1.8 & +1.4 & +1.3 & $\begin{array}{c}-11 \\
-8.3\end{array}$ & $\begin{array}{l}+5.3 \\
+1.1\end{array}$ & $\begin{array}{l}-5.6 \\
-5.9\end{array}$ & $\begin{array}{l}+13 \\
+6.7\end{array}$ & $\begin{array}{l}-11 \\
-10\end{array}$ & $\begin{array}{l}-6.7 \\
-6.7\end{array}$ & $\begin{array}{l}\mathrm{S} \\
\mathrm{S}\end{array}$ \\
\hline Mean & +4.0 & +3.9 & +2.0 & +1.8 & +1.4 & +1.3 & -9.7 & +3.2 & -5.8 & +9.9 & -10.5 & -6.7 & \\
\hline
\end{tabular}

+ : Hypermetropia, - : Myopia, 0: Emmetropia, S : Severing optic nerve, At : Atropine injection, $\mathrm{Cu}$ : Curare injection, $\mathrm{Am}$ : Amelizol injection, $\mathrm{K}$ : Killing by laceration of heart.

b. In the fishes which show the highest myopia in the fore direction

\begin{tabular}{|c|c|c|c|c|c|c|c|c|c|c|c|c|c|}
\hline & & $\mathrm{P}$ & $r e-t$ & ent & & & & & ost-tr & atment & & & \\
\hline $\begin{array}{l}\text { Species } \\
\text { (Body } \\
\text { length cm) }\end{array}$ & $\begin{array}{l}3 \\
0 \\
0\end{array}$ & 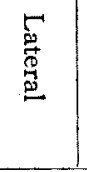 & 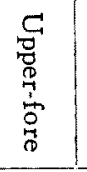 & 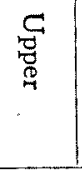 & 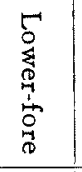 & $\begin{array}{l}5 \\
0 \\
\vdots \\
9 \\
1\end{array}$ & 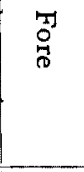 & 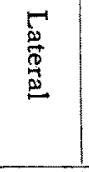 & 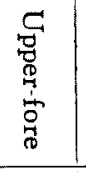 & 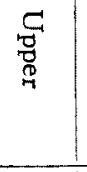 & $\begin{array}{l}5 \\
0 \\
0 \\
9 \\
0 \\
0 \\
0\end{array}$ & $\underset{5}{5}$ & 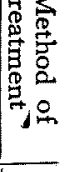 \\
\hline $\begin{array}{l}\text { pine phelus } \mathrm{L} \text {. } \\
\text { ptemfas- R. } \\
\text { iatus (14) }\end{array}$ & $\begin{array}{l}+7.2 \\
+5.6\end{array}$ & $\begin{array}{l}+4.3 \\
+4.8\end{array}$ & $\begin{array}{l}5.9 \\
5.9\end{array}$ & $\begin{array}{l}+5.6 \\
+5.6\end{array}$ & $\begin{array}{l}+4.3 \\
+5.0\end{array}$ & $\begin{array}{l}+4.3 \\
+4.8\end{array}$ & $\begin{array}{l}-17 \\
-14\end{array}$ & $\begin{array}{l}-5.9 \\
-5.6\end{array}$ & $\begin{array}{l}-6.7 \\
-5.6\end{array}$ & $\begin{array}{l}+5.9 \\
+5.0\end{array}$ & $\begin{array}{l}-5.0 \\
-4.8\end{array}$ & $\begin{array}{l}0 \\
0\end{array}$ & S \\
\hline " (15) $\frac{\mathrm{L} .}{\mathrm{R} .}$ & $\begin{array}{l}+3.7 \\
+3.7\end{array}$ & $\begin{array}{l}+3.7 \\
+5.0\end{array}$ & $\begin{array}{l}+4.0 \\
+3.7\end{array}$ & $\begin{array}{l}+4.0 \\
+3.7\end{array}$ & $\begin{array}{l}+3.5 \\
+3.3\end{array}$ & $\begin{array}{l}+3.3 \\
+3.0\end{array}$ & $\begin{array}{l}-13 \\
-13\end{array}$ & $\begin{array}{l}+3.5 \\
+5.0\end{array}$ & $\begin{array}{l}-5.6 \\
-7.7\end{array}$ & $\begin{array}{l}+5.3 \\
+5.0\end{array}$ & $\begin{array}{l}-5.9 \\
-5.6\end{array}$ & $\begin{array}{l}+5.6 \\
+7.7\end{array}$ & $\mathrm{~S}$ \\
\hline " (14) $\stackrel{\mathrm{L} .}{\mathrm{R} .}$ & $\begin{array}{l}+8.3 \\
+5.3\end{array}$ & $\begin{array}{l}+4.3 \\
+3.9\end{array}$ & $\begin{array}{l}+4.3 \\
+4.2\end{array}$ & $\begin{array}{l}+4.0 \\
+4.0\end{array}$ & $\begin{array}{l}+4.2 \\
+3.5\end{array}$ & $\begin{array}{l}+4.0 \\
+3.3\end{array}$ & $\begin{array}{l}-14 \\
-13\end{array}$ & $\begin{array}{l}+4.0 \\
+4.8\end{array}$ & $\begin{array}{l}-7.7 \\
-9.1\end{array}$ & $\begin{array}{l}-7.7 \\
-9.1\end{array}$ & $\begin{array}{l}-5.0 \\
-5.3\end{array}$ & $\begin{array}{l}+3.5 \\
+5.9\end{array}$ & $S$ \\
\hline lean & +5.6 & +4.3 & +4.7 & +4.5 & +4.0 & +3.8 & -14 & +1.0 & -7.1 & +0.7 & -5.3 & +3.8 & \\
\hline $\begin{array}{c}\text { ebastiscus } \mathrm{L} \text {. } \\
\text { (18) }\end{array}$ & $\begin{array}{l}+2.0 \\
+2.0\end{array}$ & $\begin{array}{l}+2.8 \\
+3.2\end{array}$ & $\begin{array}{l}+2.2 \\
+4.2\end{array}$ & $\begin{array}{l}+2.1 \\
+3.9\end{array}$ & $\begin{array}{l}+1.0 \\
+1.1\end{array}$ & $\begin{array}{c}0 \\
+0.6\end{array}$ & $\begin{array}{l}-13 \\
-11\end{array}$ & $\begin{array}{l}+0.7 \\
+0.9\end{array}$ & $\begin{array}{l}-6.7 \\
-6.3\end{array}$ & $\begin{array}{l}+2.4 \\
+1.1\end{array}$ & $\begin{array}{l}-5.9 \\
-5.6\end{array}$ & $\begin{array}{l}-2.5 \\
+0.9\end{array}$ & $\begin{array}{l}S \\
S\end{array}$ \\
\hline
\end{tabular}




\begin{tabular}{|c|c|c|c|c|c|c|c|c|c|c|c|c|c|}
\hline$"$ (18) & +4.2 & +4.0 & & & & & -13 & +3.3 & & & & & At \\
\hline$" \quad(14)$ & +2.3 & +3.5 & +3.3 & $3+2.5$ & +1.3 & 0 & -11 & +2.5 & -7.7 & +2.0 & -6.7 & 0 & At \\
\hline (16) & +2.2 & +2.6 & & & & & -13 & +2.0 & & & & & $\mathrm{~S}$ \\
\hline (15) & +2.6 & +3.1 & & & & & -13 & +2.2 & & & & & $\mathrm{~S}$ \\
\hline Mean & +2.6 & +3.2 & +3.2 & +2.8 & +1.1 & +0.2 & $\mid-12.3$ & +1.9 & -6.9 & +1.8 & -6.1 & -0.5 & \\
\hline $\begin{array}{l}\text { Therapon } \\
\text { oxyrhynchus } \\
\qquad(20)\end{array}$ & +3.3 & +4.3 & +2.0 & & +2.7 & & -14 & 0 & -10 & & -6.7 & & S \\
\hline $\begin{array}{l}\text { Seriola quin- } \\
\text { queradiata (17) }\end{array}$ & +3.3 & +8.3 & +3.3 & $3+11$ & +4.0 & +6.3 & -12 & +9.1 & $|-7.1|$ & +5.6 & -8.3 & +7.7 & At \\
\hline $\begin{array}{l}\text { Girella } \\
\text { punctata }\end{array}$ & +5.6 & +3.9 & +5.0 & +3.3 & +2.0 & 0 & -14 & +4.0 & -6.7 & +3.6 & -6.3 & 0 & At \\
\hline (18) & +5.9 & +3.7 & +5.0 & & +5.6 & & -13 & +3.3 & -7.2 & & -8.3 & & $\mathrm{~K}$ \\
\hline Mean & +5.8 & +3.8 & +5.0 & $\mid+3.3$ & +3.8 & 0 & $|-13.5|$ & +3.7 & -7.0 & +3.6 & -7.3 & 0 & \\
\hline $\begin{array}{l}\text { Pseudoblennius } \\
\text { totomius ( } 7.5)\end{array}$ & +5.9 & +7.7 & +6.3 & +6.7 & +7 & +13 & -25 & +7.7 & -10 & +8.3 & -8.3 & +14 & $\mathrm{~S}$ \\
\hline
\end{tabular}

c. In the fishes which show the highest myopia in the upper-fore direction

\begin{tabular}{|c|c|c|c|c|c|c|c|c|c|c|c|c|c|}
\hline \multirow{2}{*}{$\begin{array}{l}\text { Direction } \\
\text { Species } \\
\text { (Body } \\
\text { length } \mathrm{cm})\end{array}$} & \multicolumn{6}{|c|}{ Pre-treatment } & \multicolumn{6}{|c|}{ Post-treatment } & \multirow[b]{2}{*}{ 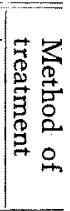 } \\
\hline & $\begin{array}{l}7 \\
0 \\
0\end{array}$ & 占 & 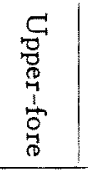 & $\underset{\substack{0 \\
⿱ 乛 龰}}{C}$ & $\begin{array}{l}5 \\
0 \\
8 \\
0 \\
0 \\
0 \\
0 \\
0\end{array}$ & 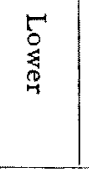 & $\begin{array}{l}3 \\
0 \\
0 \\
0\end{array}$ & 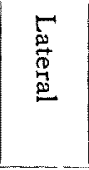 & $\begin{array}{l}c \\
5 \\
0 \\
0 \\
1 \\
0 \\
0 \\
0 \\
0\end{array}$ & $\stackrel{C}{c}$ & $\begin{array}{l}5 \\
5 \\
\vdots \\
0 \\
1 \\
0 \\
0 \\
0 \\
0\end{array}$ & $\begin{array}{l}5 \\
0 \\
\vdots \\
0\end{array}$ & \\
\hline $\begin{array}{ll}\text { ateolabrax } \\
\text { aponicus } & \mathrm{L} . \\
\mathrm{R}\end{array}$ & $\begin{array}{l}+5.3 \\
+5.9\end{array}$ & $\begin{array}{l}+4.8 \\
+5.6\end{array}$ & & & & & $\begin{array}{l}-13 \\
-13\end{array}$ & $\begin{array}{l}+5.3 \\
+5.6\end{array}$ & $\begin{array}{l}-13 \\
-14\end{array}$ & $\begin{array}{l}-8.3 \\
-10\end{array}$ & $\begin{array}{l}-6.7 \\
-7.7\end{array}$ & $\begin{array}{l}+13 \\
+11\end{array}$ & $\begin{array}{l}S \\
S\end{array}$ \\
\hline " (18.5) $\stackrel{\mathrm{L} .}{\mathrm{R} .}$ & $\begin{array}{l}+7.2 \\
+6.7\end{array}$ & $\begin{array}{l}+6.3 \\
+5.9\end{array}$ & $\begin{array}{l}+4.8 \\
+5.0\end{array}$ & $\begin{array}{l}+4.8 \\
+5.0\end{array}$ & $\begin{array}{l}+2.5 \\
+2.3\end{array}$ & $\begin{array}{l}0 \\
0\end{array}$ & $\begin{array}{l}-11 \\
-14\end{array}$ & $\begin{array}{l}+5.6 \\
+5.3\end{array}$ & $\begin{array}{l}-13 \\
-14\end{array}$ & $\mid \begin{array}{c}-8.3 \\
-11\end{array}$ & $\begin{array}{l}-5.0 \\
-4.3\end{array}$ & $\begin{array}{l}+8.3 \\
+8.3\end{array}$ & S \\
\hline " (19) $\quad \stackrel{\mathrm{L}}{\mathrm{R}}$. & $\begin{array}{l}+5.6 \\
+5.9\end{array}$ & $\begin{array}{l}+5.0 \\
+4.3\end{array}$ & $\begin{array}{l}+4.8 \\
+4.3\end{array}$ & $\begin{array}{l}+4.8 \\
+4.0\end{array}$ & $\begin{array}{l}+2.5 \\
+1.7\end{array}$ & $\begin{array}{l}+2.5 \\
+1.7\end{array}$ & $\begin{array}{l}-14 \\
-13\end{array}$ & $\begin{array}{l}+5.0 \\
+4.8\end{array}$ & $\begin{array}{l}-14 \\
-13\end{array}$ & $\begin{array}{l}+4.0 \\
+4.2\end{array}$ & $\begin{array}{l}-5.6 \\
-5.0\end{array}$ & $\begin{array}{l}+2.2 \\
+3.0\end{array}$ & $\mathrm{~S}$ \\
\hline " (23) & +7.2 & +5.9 & +7.7 & +7.7 & +7.2 & +7.2 & -14 & +5.9 & -14 & -9.1 & -7.7 & +25 & $S$ \\
\hline$" \quad(9.5)$ & +7.7 & +9.1 & & & & & -17 & +13 & -20 & -14 & -10 & +25 & S \\
\hline 11 (17) & +5.0 & & & & & & -11 & & -12 & & & & Am \\
\hline$\prime \prime(17.5)$ & +4.8 & & & & & & -13 & & -13 & & & & Am \\
\hline$\prime \prime(17.5)$ & +2.9 & & & & & & -13 & & -13 & & & & At \\
\hline " (18) & +7.2 & +4.8 & +6.7 & +5.0 & 0 & 0 & -9.1 & +3.3 & -10 & 0 & -8.7 & +2.5 & $\mathrm{Cu}$ \\
\hline Mean & +6.0 & +5.7 & +5.6 & +5.2 & +2.7 & +1.9 & -12.9 & +6.0 & -13.6 & -5.8 & -6.7 & +10.8 & \\
\hline $\begin{array}{l}\text { Trachurus } \\
\text { japonicus } \\
\text { (10.5) }\end{array}$ & 0 & +5.9 & & & & & -15 & +5.9 & -20 & -10 & +3.3 & +6.7 & At \\
\hline " (10.5) & +2.2 & +5.0 & +1.8 & +1.8 & +1.8 & +1.8 & -17 & +5.0 & -20 & -6.7 & +2.9 & +7.7 & At \\
\hline Mean & +1.1 & +5.5 & +1.8 & +1.8 & +1.8 & +1.8 & -16 & +5.5 & -20 & -8.4 & +3.1 & +7.2 & \\
\hline
\end{tabular}

assumed to occur between the five treatments. In Table 7 a the greatest myopia occurred in the lower-fore direction. In Table $7 \mathrm{~b}$ it occurred in the fore direction and in Table $7 \mathrm{c}$ in the upper-fore direction. Obviously the lenses must have been displaced in these respective directions, and it will be shown in the section of visual axis that the direction 
of displacement in these fishes coincide with their visual axes as established by another method.

The results of these refraction measurements are not directly comparable with BEER's. In this report, the pre-treatment state in assumed to be the resting state of the eye, where BEER assumed the resting state to be that produced by the injection of curare or atropine. Further-more, the degree of myopia obtained by BEER does not necessarily reflect the highest myopia of the fish, because he consistently measured refraction along only one line, i.e., a line passing through the center of the lens to a point near the scotoma*. This, perhaps, coincides with the lateral-fore direction in Fig. 6. His values of myopia ranged from -3 to -10 diopters, and -12 in one extreme case (mean -6.1 ), while the values in Table 7 range from -10 to -25 diopters (mean -14 ).

According to Table 7, the accommodation is assumed to be performed along certain directions, except which the refraction is hypermetropic or emmetropic in either pre- or post-treatment state. It would be of little importance if the fish eye is myopic, emmetropic or hypermetropic in the resting state, because the movement perception, for which the acute image is not neccessary, might play more important part in detecting the food or the enemy as Tamura (1952) showed in young Lateolabrax japonicus. So as to face the interesting moving object which is perceived at first by its movement, the fish bends or turns its head and body and keeps it in the visual axis which is, in general, situated in the binocular field, and then the clear from perception, for which the accommodation and acute image are indispensable, becomes to play an important part to discern the object.

\section{Visual axis}

In general, the visual axis can be defined as the line of most acute vision, and in foveate animals it passes throught the fovea and center of the lens. In animals lacking a fovea, but having an area of high cone density, a line from the center of the area through the center of the lens may be considered the visual axis. Since the fish examined in this study do not have foveae, the latter definition applies. Thus the fishes with area in the dorso-temporal, temporal and ventro-temporal have visual axes extending, respectively, into the lower-fore, fore, and upper-fore regions of the visual field. It may further be assumed that the line of greatest accommodation will coincide with the visual axis.

Table 8 compares these two sets of data (taken from Tables 5 and 7) for 14 species. Among them, cone density of Pseudoblennius was not measured, but a typical fovea could be seen in the temporal region of retina. Columns 1 and 2 show the regions of highest and second highest cone density, while columns 3 and 4 snow the directions of highest and second highest accommodation. Agreement between the two sets of observations is almost perfect. The direction of the visual axis is noted in column 5 .

Girella and Lateolabrax are the only two cases showing disagreement. In Girella, this is readily explained by negligible differences in cone uensity between the ventrotemporal and temporal regions. Assuming them to be the same, the direction of the axis is determined by the line of greatest accommodation (Fore). In Lateolabrax there is a

* Pigment auf der Papille, Ausstrahlung des Sehnerven, Arterie des Processus falciformis etc. 
Table 8. Direction of visual axis

\begin{tabular}{|c|c|c|c|c|c|}
\hline \multirow[b]{2}{*}{ Species } & \multicolumn{2}{|c|}{ After Table 5} & \multicolumn{2}{|c|}{ After Table 7} & \multirow[b]{2}{*}{$\begin{array}{l}\text { Direction of } \\
\text { Visual axis de- } \\
\text { cided from left } \\
\text { columns }\end{array}$} \\
\hline & $\begin{array}{l}\text { Retinal region } \\
\text { of highest de- } \\
\text { nsity of cones } \\
\text { (No. of cones) }\end{array}$ & $\begin{array}{l}\text { Retinal region } \\
\text { of second hi- } \\
\text { ghest density } \\
\text { of cones (No. } \\
\text { of cones) }\end{array}$ & $\begin{array}{l}\text { Direction of } \\
\text { highest myo- } \\
\text { pia (Refracti- } \\
\text { tion in Diop.) }\end{array}$ & $\begin{array}{l}\text { Direction of } \\
\text { second high- } \\
\text { est myopia } \\
\text { (Refraction } \\
\text { in Diop.) } \\
\end{array}$ & \\
\hline Pagrosomus major & $\begin{array}{l}\text { Dorso- } \\
\text { temporal } \\
(303)\end{array}$ & $\begin{array}{l}\text { Dorsal } \\
(240)\end{array}$ & $\begin{array}{l}\text { Lower-fore } \\
(-16.5)\end{array}$ & $\begin{array}{l}\text { Fore } \\
(-10.5)\end{array}$ & Lower-fore \\
\hline Sparus hasta & $\begin{array}{l}\text { Dorso- } \\
\text { temporal } \\
(400)\end{array}$ & $\begin{array}{l}\text { Temporal } \\
(147)\end{array}$ & $\begin{array}{l}\text { Lower fore } \\
(-13.0)\end{array}$ & $\begin{array}{l}\text { Fore } \\
(-11.2)\end{array}$ & Lower-fore \\
\hline Evynnis japonicus & $\begin{array}{l}\text { Dorso- } \\
\text { temporal } \\
(570)\end{array}$ & $\begin{array}{l}\text { Temporal } \\
(393)\end{array}$ & $\begin{array}{l}\text { Lower-fore } \\
(-13.0)\end{array}$ & $\begin{array}{l}\text { Fore } \\
(-11.8)\end{array}$ & Lower-fore \\
\hline Leiognathus argenteus & $\begin{array}{l}\text { Dorso- } \\
\text { temporal } \\
(236)\end{array}$ & $\begin{array}{l}\text { Temporal } \\
(209)\end{array}$ & $\begin{array}{l}\text { Lower fore } \\
(-14.0)\end{array}$ & $\begin{array}{l}\text { Fore } \\
(-14.0)\end{array}$ & Lower-fore \\
\hline Xesurus scalprum & $\begin{array}{l}\text { Dorso- } \\
\text { temporal } \\
(252)\end{array}$ & $\begin{array}{l}\text { Ventro- } \\
\text { temporal } \\
(236)\end{array}$ & $\begin{array}{l}\text { Lower-fore } \\
(-10.5)\end{array}$ & $\begin{array}{l}\text { Fore }^{\prime} \\
(-9.7)\end{array}$ & Lower-fore \\
\hline $\begin{array}{l}\text { Epinephelus septem- } \\
\text { fasciatus }\end{array}$ & $\begin{array}{l}\text { Temporal } \\
(1050)\end{array}$ & $\begin{array}{l}\text { Nasal } \\
(253)\end{array}$ & $\begin{array}{l}\text { Fore } \\
(-14.0)\end{array}$ & $\begin{array}{l}\text { Upper fore } \\
(-7.1)\end{array}$ & Fore \\
\hline Sebast iscus marmoratus & $\begin{array}{l}\text { Temporal } \\
(380)\end{array}$ & $\begin{array}{l}\text { Dorso- } \\
\text { temporal } \\
(105)\end{array}$ & $\begin{array}{l}\text { Fore } \\
(-12.3)\end{array}$ & $\begin{array}{l}\text { Upper-fore } \\
(-6.9)\end{array}$ & Fore \\
\hline Therapon ox yrh ynchus & $\begin{array}{l}\text { Temporal } \\
(133)\end{array}$ & $\begin{array}{l}\text { Nasal } \\
(93)\end{array}$ & $\begin{array}{l}\text { Fore } \\
(-14)\end{array}$ & $\begin{array}{l}\text { Upper-fore } \\
(-10)\end{array}$ & Fore \\
\hline Seriola quinqueradiata & $\begin{array}{l}\text { Temporal } \\
(292)\end{array}$ & $\begin{array}{l}\text { Ventral } \\
(264)\end{array}$ & $\begin{array}{l}\text { Fore } \\
(-12)\end{array}$ & $\begin{array}{l}\text { Lower-fore } \\
(-8.3)\end{array}$ & Fore \\
\hline Girella punctata & $\begin{array}{l}\text { Ventro- } \\
\text { temporal } \\
(244)\end{array}$ & $\begin{array}{l}\text { Temporal } \\
(237)\end{array}$ & $\begin{array}{l}\text { Fore } \\
(-13.5)\end{array}$ & $\begin{array}{l}\text { Lower-fore } \\
(-7.3)\end{array}$ & (Fore) \\
\hline Pseudoblennius totomius & $\begin{array}{l}\text { Fovea exists in } \\
\text { ral region of }\end{array}$ & $\begin{array}{l}\text { n the tempo- } \\
\text { retina }\end{array}$ & $\begin{array}{l}\text { Fore } \\
(-25)\end{array}$ & $\begin{array}{l}\text { Upper-fore } \\
(-10)\end{array}$ & Fore \\
\hline Lateolabrax japonicus & $\begin{array}{l}\text { Temporal } \\
(259)\end{array}$ & $\begin{array}{l}\text { Ventro- } \\
\text { temporal } \\
(158)\end{array}$ & $\begin{array}{l}\text { Upper-fore } \\
(-13.6)\end{array}$ & $\begin{array}{l}\text { Fore } \\
(-12.9)\end{array}$ & $\begin{array}{l}\text { (Fore-upper- } \\
\text { fore) }\end{array}$ \\
\hline Trachurus japonicus & $\begin{array}{l}\text { Ventro- } \\
\text { temporal } \\
(288)\end{array}$ & $\begin{array}{l}\text { Ventral } \\
(272)\end{array}$ & $\begin{array}{l}\text { Upper-fors } \\
(-20)\end{array}$ & $\begin{array}{l}\text { Fore } \\
(-16)\end{array}$ & Upper-fore \\
\hline
\end{tabular}

difference in the density between the temporal and ventro-temporal regions, but temporal region, which has the highest density, covers a broad area. Also, accommodation differs by only 0.7 of a diopter between the upper-fore and fore directions, so the visual axis is fore-upper-fore, a compromise between the two.

The relative positions of the ligamentum suspensorium and retractor lentis in respect to the lens offers a third means of estimating the visual axis. Three arrangements were found in the fishes examined (Fig. 9). The fishes listed in Table 7 a have the ligament attachment on the naso-dorsal surface of the lens and the muscle attachment on the ventrotemporal surface of the lens. Contraction of the muscle would move the lens up and back towards the dorso-temporal region of the retina. This is consistent with other evidence that the visual axis in this group is lower-fore in direction. Similarly, the fishes in Table $7 \mathrm{~b}$ have the ligament attached dorsally and the muscle ventrally, indicating tnat 
the lens accommodates on the fore axis; those in Table $7 \mathrm{c}$ have the ligament attached dorso-temporally and the muscle attached ventronasally, indicating lens movement along the upper-fore axis.

Direction of accommodation was estimated in this way for eight species where live specimens could not be obtained for optical methods.

Fig. 10 shows that Cantherines modestus, Helicolenus dactylopterus, and Scomber japonicus probably move the lens along the fore axis, whereas Priacanthus boops, Malakichthys wakiyae, Zenion japonicum, Argentina semifasciata and Chlorophthalmus albatrossis probably move it along the upper- upperfore axis. This is consistent with the topography of the retina, for the first group has the highest cone density in the temporal region, and the second group has the highest cone density in the ventral region.

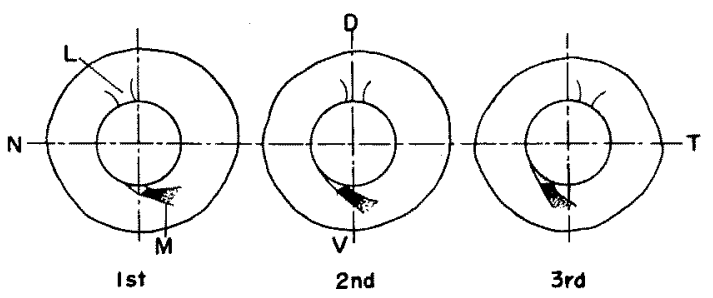

Fig. 9. Three types of attaching points.

S : Suspensory ligament, M : Retractor muscle.

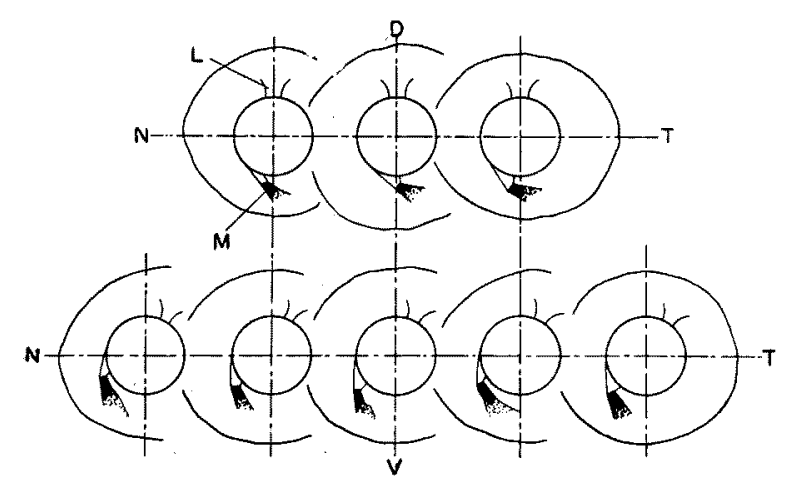

Fic. 10. The attaching point in some fishes.

From left to right ; upper row, Cantherines modestus, Helicolenus dact ylopterus and Scomber japonicus; lower row, Priacanthus boops, Malakichthys wakiyae, Zenion japonicum, Argentina semifasciata and Chlo- $^{-}$ rophthalmus albatrossis.

The ecological significance of different visual axes is quite obvious when one considers the feeding behaviour of the various fish that have been examined. Pagrosomns, Sparus, Evynnis, Leiognathus and Xesurus, which have lower-fore visual axes, consistently picked over the bottom of the aquarium in search of food. They are generally classed as bottom feeders. Epinephelus, Sebastiscus, and Pseudoblennis, which have somewhat conspicuous areae temporales and fore visual axes, display a tendency to take food in front of them. These fish are known to live in among rocks and seaweed in nature, where they attack small animals that pass by them. It was also noted in the aquarium that their eyes have considerable scope of movement and are of ten focused simultaneously on objects in front of them, indicating the importance of binocular vision to them. Aquarium observations were inadequate on Therapon, Cantherines, Chelidonichthys, Sphaeroides, Seriola, and Girella, which also have fore visual axes. Labeolabrax, Trachurus and Priacanthus, judged to have upper-fore axes, tended to take food ahead and above them in the aquarium. This was particularly true for Priacanthus, which ordinarily paid no attention to food of not moving unless it was in the upper-fore or upper portion of the visual field.

It must not be forgotten, however, that the entire monocular field, usually about $180^{\circ}$, is quite important to all fish. Peripheral perception of movement for which high 
acuity is not essential facilitates their detection of prey and predator over the entire visual field.

\section{Binocular visual field}

The binocular field, which is present to a greater or lesser extent in most fishes, is formed by the the anterior overlap of the wide monocular fields. Its width is perhaps indicative of its functional significance. In general, fish that live among rocks and seaweeds in the littoral zone and prey on other small organisms have the wider fields. However, it must be remembered that the field is not rigidly fixed, since the eye of fish rotate independently. In the present study, the width of the binocular field has been measured at eight elevations.

Method : Specimens were anaesthetized and fixed in a transparent chamber so that they could be rotated around the horizontal and vertical axes (Fig. 11). The eyes were then viewed through the same ophthalmoscope with which refractions were measured. Any point from which both eyes reflected light was assumed to be in the binocular field. At each elevation the fish was rotated clockwise around the vertical axis until the limit of reflection was reached in the rignt eye. The fish was then rotated counterclockwise until the limit was reached in the left eye. From the included angle $(\beta)$, the distance between the vertical axis and the eyes of the fish $(l)$, the interorbital distance $(d)$, and the distance between the observation point and vertical axis $(L)$, the width of the bino-
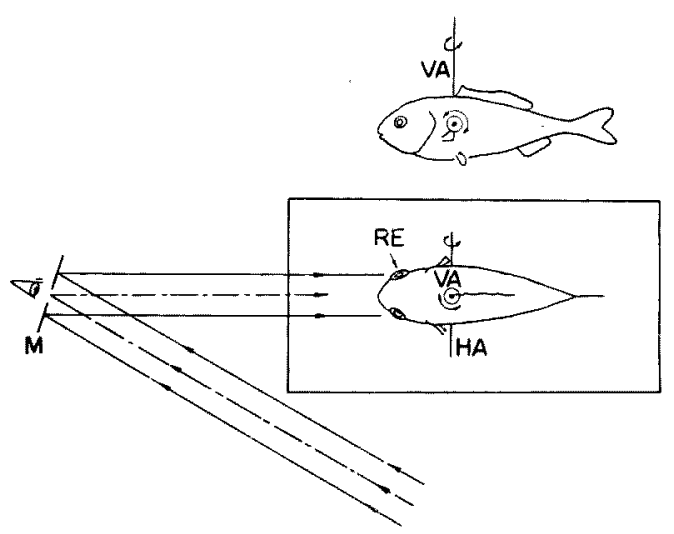

Fig. 11. Schema showing the method of measuring the extent of binocular field.

M : Mirror, VA : Vertical axis, HA : Horizontal axis, RE : Right eye of the fish. cular field was found by drawing a figure like Fig. 12.

Results : The results are clearly summarized in Table 9. All the species examined have definite binocular fields. Although the data indicate that the field covers a rather broad vertical range, it is noteworthy that the widest angle, in eacn case, is aligned with the visual axis.

\section{Summary and Conclusion}

1. Three properties of the eye which are importand in visual acuity were investigated : the resolving power of the dioptric system, the resolving power of

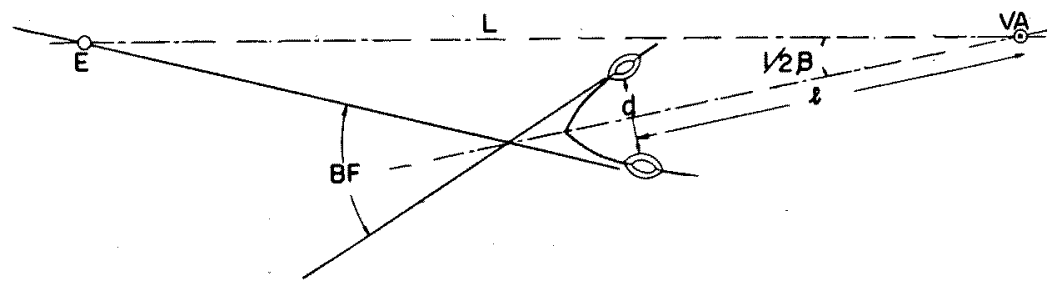

Fig. 12. The method of finding the binocular field (BF) from the rotated angle ( $\beta$ ) etc. $\mathrm{E}$ : Observation point, VA : Vertical axis. 
Table 9. Width of the binocular field at various elevations

\begin{tabular}{|c|c|c|c|c|c|c|c|c|c|}
\hline \multirow{2}{*}{ Species } & \multirow{2}{*}{$\begin{array}{l}\text { Direction of visual } \\
\text { axis }\end{array}$} & \multicolumn{8}{|c|}{ Elevation of visual direction } \\
\hline & & $-60^{\circ}$ & $-40^{\circ}$ & $-20^{\circ}$ & $0^{\circ}$ & $20^{\prime}$ & $30^{\circ}$ & $40^{\circ}$ & $60^{\circ}$ \\
\hline Pagrosomus major & Lower fore & $\longrightarrow$ & $26^{\circ}$ & $28^{\circ}$ & $24^{\circ}$ & $20^{\circ}$ & $18^{\circ}$ & $10^{\circ}$ & $14^{\circ}$ \\
\hline Evynnis japonicus & Lower-fore & - & 24 & 26 & 22 & 19 & 15 & 10 & 12 \\
\hline Sparus hasta & Lower-fore & $10^{*}$ & 25 & 26 & 21 & 17 & 15 & 10 & 13 \\
\hline Leiognathus argenteus & Lower-fore & 19 & 18 & 23 & 18 & 15 & - & 9 & 6 \\
\hline Xesurus scalprum & Lower-fore & 1 & 12 & 15 & 15 & 13 & - & 13 & 12 \\
\hline Epine phelus septemfasciatus & Fore & - & 15 & 17 & 32 & 28 & - & 20 & - \\
\hline Sebastiscus marmoratus & Fore & - & - & 20 & 34 & 28 & - & 20 & 6 \\
\hline Therapon oxyrhynchus & Fore & $一$ & - & 23 & 30 & 27 & - & 18 & 一 \\
\hline Lateolabrax japonicus & For upper-fore & - & - & 8 & 12 & 17 & 20 & 19 & 12 \\
\hline Trachurus japonicus & Upper-fore & $-\cdots$ & - & 13 & 15 & 22 & 23 & 20 & 18 \\
\hline
\end{tabular}

$\mathrm{O}$ is in the horizontal plane, negative angles below it and positive angles above it. The direction of the visual axis is taken from Table 8 .

the retina, and the extent of accommodation.

2. The resolving power of the crystalline lens, the only dioptric element responsible for image formation in fishes, ranged 54-90 seconds of arc for lenses over about $5 \mathrm{~mm}$ in diameter.

3. Cone density varies by species and by regions of retina. The best retinal acuity was 4.2 minutes of arc, so that the retina, not the lens, limits visual acuity.

4. All eyes examined were either emmetropic or hypermetropic in untreated living fish which was fixed rigidly in a tank between two pieces of rubber sponge, but became myopic in certain directions upon injection of atropine or curare, or upon sectioning of the optic nerve.

5. The axis of greatest accommodation was lower-fore in Pagrosomus, Sparus, Evynnis, Leiognathus and Xesurus; fore in Epinephelus, Sebastiscus, Therapon, Girella and Pseudoblennius; and fore-fore-upper or upper-fore in Lateolabrax and Trachurus. These axes are usually served by the retinal region of highest cone density.

6. There is a relationship between attachment points of the suspensory ligament and retractor lentis and the axis of accommodation. From this relationship and the region of highest cone density, the visual axis was determined for some species not available in living condition. They are : fore in Cantherines, Helicolenus, and Scomber ; upper-upper -fore in Priacanthus, Malakichthys, Zenion, Chlorophthalmus and Argentina.

7. The binocular field tends to be broadest in the direction of the visual axis.

\section{Ref erences}

Beer, T., 1894. Die Accommodation des Fischauges. Pflüg. Arch., 58 : 523-650.

BRUnNer, G., 1934. Über die Sehscharfe der Elritze bei verschiedenen Heilligkeiten. $Z$. vergl. Physiol., $21: 296-316$.

Friscr, K. von, 1925. Farbensinn der Fische und Duplizitäts-theorie. ibid., 2:393-452.

Harms, J.W., 1914. Über die Augen der am Grunde der Gewässer lebenden Fische. Zool. Anz. $44: 35-41$.

Herter, K., 1930. Weitere Dressurversuche an Fischen. Z. vergl. Physiol., $11: 730-748$. 
Hess, C. von, 1912. Vergleichende Physiologie des Gesichtssinnes. Handb. vergl. Physiol. (ed. WINTERSTEIN), $4: 1-269$.

Hogben, L. \& Landgrebe, F., 1940. The pigmentary effector system. X. The receptor fields of the teleostean visual response. Proc. Roy. Soc. B, $128: 317-342$.

Kammann, H., 1934. Über das Vorkommen einer Fovea centralis in Knochenfischauge. Zool. Anz., $106: 49-56$.

KammanN, H., 1936. Über das foveale Sehen der Wirbeltiere (I. Über die Fovea centralis und Fovea lateralis bei einigen Wirbeltieren). von Graefe's Arch. Ophthal., 135 : 265-276.

Matthimssen, L., 1882. Über die Beziehung, welche zwischen dem Brechungsindex des Kerncentrums der Krystallinse und dem Dimension des Auges bestehen. Pflüg. Arch., $27: 510-523$.

Meader, R.G., 1936. Accommodation and its reflex pathways in the Teleosts. Yale J. Biol. Med., $8: 511-522$. (from Biol. Abst.)

Mülcter, H., 1952. Bau und Wachstum der Netzhaut des Guppy (Lebistes reticulatus). Zool. Jahrb., $63: 275-324$,

Patten, B. M. and R. Philpotr, 1921. The shrinkage of embryos in the processes preparatory to sectioning. Anat. Rec., $20: 393-413$.

Polyak, S. L., 1948. The retina. University of Chicago Press : 1-605.

Takatsuzr, M., 1939. Die Sehzellen in der Netzhaut der Fisch, besonders ihre Reiheordnungen. Kaibogaku zassi, $14: 1-68$.

TamURA, T., 1952. On the senses of food-searching in Lateolabrax japonicus. Bull. Jap. Soc. Sci. Fish., $17: 296-300$.

Walts, G.L., 1942. The vertebrate eye and its adaptive radiation. Cranbrook Ins. of Science, Bull. $19: 1-785$.

Wonder, W., 1936. Physiologie der Susswasserfische Mitteleuropas. Handb. der Binnenfischerei. Mitteleuropas (ed. Denoll-Maler), II B: $1-340$.

Yамамото, S., 1931. Die statische Refraktion des Auges verschiedener Tiere. Okayama-IgakkaiZasshi, $43: 1407-1425$.

Yамамото, Т., 1949. Dobutu seiri no zikken. Tokyo., 1-212.

Yamanodchi, T., 1940. Gyo-rui no si-ryoku (siseido) ni tuite. (Summary of oral lecture). Zool. Mag. , $38: 75$. 